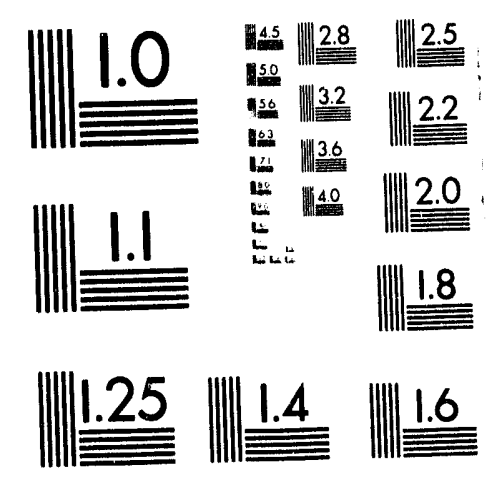



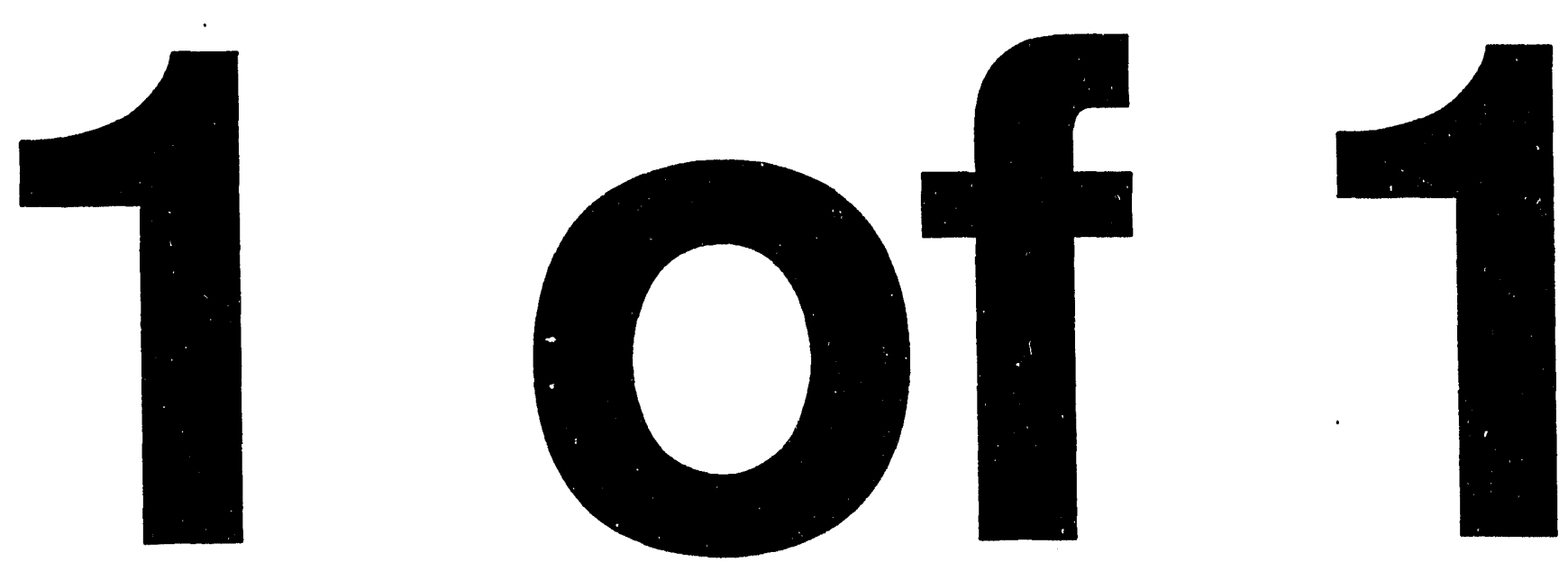


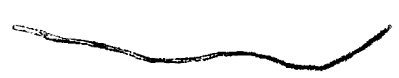

$$
\begin{gathered}
\text { ARGONNE NATIONAL LABORATORY } \\
9700 \text { South Cass Avenue } \\
\text { Argonne, Illinois } 60439
\end{gathered}
$$

ANL/CNSV-TM-135

\begin{abstract}
A SUMMARY OF TRUCK FUEL-SAVING MEASURES DEVELOPED WITH INDUSTRY PARTICIPATION
\end{abstract}

by

\author{
K.M. Bertram, C.L. Sar1cks, E.W. Gregory II,* and A.J. Moore** \\ Energy and Environmental Systems Division \\ Center for Transportation Research
}

September 1983

work sponsored by

U.S. DEPARTMENT OF ENERGY

Assistant Secretary for Conservation and Renewable Energy office of Vehicle and Engine Research and Development

*U.S. Department of Energy.

**Northwestern University, Evanston, Ill. 
SUMMARY.............................................. v

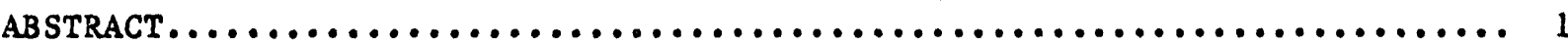

1 INTRODUCTION..............................................

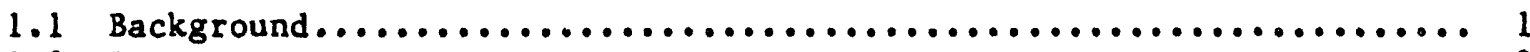

1.2 Purpose...............................................

2 METHODS...............................................

2.1 Literature Review...................................... 3

2.2 Development of Draft Matrix.............................. 3

2.3 Industry Coordination Meeting and Revision of Matrix............

$2: 4$ Press Release and Distribution of Matrix.....................5

2.5 Report Preparation and Distribution........................

3 DESCRIPTION OF MATRIX..................................... 6

3.1 Fuel-Saving Measures.................................... 6

3.2 Claimed Fuel Savings....................................6

3.3 Assumed Fuel Use....................................... 7

3.4 Claimed 1983 Fuel Cost Savings............................ 7

3.5 Additional 1983 Costs.................................. 7

3.6 Approximate Payback Period................................. 7

3.7 Assumed Market Sectors................................. 8

3.8 Remarks.............................................. 8

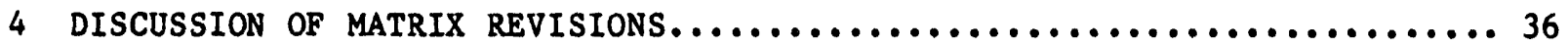

4.1 Summary of Industry Coordination Meeting Results............... 36

4.2 Addition and Deletion of Matr1x Measures.................... 37

4.3 Revisions of Fuel-Savings Percentage Estimates................. 38

4.4 Adjustments of Payback Period Estimates....................... 39

4.5 Substantive Changes to Remarks Column....................... 40

5 ConCLUSIONS............................................. 42

APPENDIX A: MATRIX REFERENCE LIST..............................45

APPENDIX B : MEETING PARTILIPANTS .............................

APPENDIX $c:$ ARGONNE PRESS RELEASE.............................

TABLE

1 Matrix of Truck Energy Efficiency Measures....................... 
This report describes the third project undertaken by the Center for Transportation Research, Argonne National Laboratory (ANL), in a U.S. Department of Energy program designed to develop and distribute compendiums of measures for saving transportation fuel. A matrix, or chart, of more than 60 fuel-saving measures was developed by ANL and refined with the assistance of trucking industry operators and researchers at an industry coordination meeting held in August 1982. The first two projects used similar meetings to refine matrices developed for the international maritime and U.S. railroad industries.

The consensus reached by those at the meeting was that the single most important element in a truck fuel-efficiency improvement program is the human element - namely the development of strong motivation among truck drivers to save fuel. The role of the driver is crucial to the successful use of fuelsaving equipment and operating procedures. Identical conclusions we re reached in the earlier maritime and rail meetings, thus providing a strong indication of the pervasive importance of the human element in energy-efficient transportation systems.

The number and variety of changes made to the matrix are also delineated, including addition and deletion of various options and revisions of fuel-saving estimates, payback period estimates, and remarks concerning items such as the advantages, disadvantages, and cautions associated with various measures. The quality and quant1ty of the suggested changes demonstrate the considerable value of using a forum of industry operators and researchers to refine research data that are intended for practical application. 


\section{A SUMMARY OF TRUCK FUEL-SAVING MEASURES \\ DEVELOPED WITH INDUSTRY PARTICIPATION}

by

K.M. Bertram, C.L. Saricks, E.W. Gregory II, and A.J. Moore

ABSTRACT

As part of a program sponsored by the U.S. Department of Energy, Argonne National Laboratory (ANL) developed a "natrix" that summarized a wide variety of fuel-saving measures, as well as assoclated costs and benefits, for the trucking industry. Information was derived primarily from technical documents and manufacturers' 11terature, but estimates of cost savings were calculated at ANL. The matrix was reviewed by trucking industry representatives at an industry coordination meeting held in 1982; revisions to the matrix were made in response to discussions at the meeting. In its final form, the matrix contained some 60 fuel-saving measures under four principal headings. Participants at the meeting agreed that the single most important factor in any effort to conserve fuel is the human element. Training and incentives are needed to develop strong motivation among drivers to save fuel. More than 2400 coples of the matrix have been distributed free of charge to members of the U.S. trucking industry.

\section{INTRODUCTION}

\subsection{BACKGROUND}

This report focuses on the third in a series of matrices, or summary charts, of information on fuel-saving measures for three major transportation industries. The first matrix was prepared for the international maritime Industry and the second was for the U.S. rallroad industry. The third, which is the subject of this report, was developed for the U.S. trucking industry. These efforts have been part of an information compliation and dissemination program conducted by the Center for Transportation Research at Argonne National Laboratory (ANL) for the office of Vehicle and Englne Research and Development in the U.S. Department of Energy.

$$
\begin{aligned}
& \text { Each of the matrices has been widely distributed. More than } 1100 \\
& \text { coples of the maritime matrix were malled to U.S. and foreign shipping }
\end{aligned}
$$


companies engaged in U.S. foreign trade. The rall matrix was sent to more than 120 U.S. railroads and to the Association of American Railroads, while the truck matrix was sent to more than 1800 members of the U.S. trucking Industry.

The response to these mallings has been very favorable. More than 100 requests for additional information on the maritime matrix were received from 30 countries, and so far about 100 requests for additional information on the truck matrix have been recelved. In addition, more than 550 requests for the matrix have been recelved from members of the trucking industry and its suppliers. International interest in the rail matrix has also been expressed. An important reason for these favorable responses has been the realism brought to the information in the matrices by the involvement of experienced industry operators. This involvement has been through participation in industry coordination meetings with the expressed purpose of refining the appropriate draft matrix as part of the project's research design.

In this report's discussion of the development and distribution of the truck matrix, special emphasis is placed on the extensive refinements made as a result of the industry coordination meeting.

\subsection{PURPOSE}

Commercial truck transportation in the U.S. consumes approximately 3 quads ( 1 quad $=10^{15} \mathrm{Btu}$ ) annually, or approximately $476 \mathrm{million} \mathrm{bb} 1 / \mathrm{yr}$. This amount, which is $15 \%$ of all transportation energy consumed annually in the U.S., is projected to consume $21.5 \%$ of annual transport energy by the year 2000. The purpose of the research described in this report is to aid the U.S. trucking industry in its efforts to use fuel more efficiently. The research involved the identification, evaluation, summarization, and distribution of useful information on fuel-saving options in order to provide the industry with a single source of concise, comprehensive data to use in selecting the most appropriate options for specific applications. 
2 METHODS

The methods used to conduct this project are as follows (listed in chronological order):

1. Literature review.

2. Development of draft matrix.

3. Industry coordination meeting and revision of matrix.

4. Press release and distribution of matrix.

5. Report preparation and distribution.

Each is described below.

\subsection{LITERATURE REVIEW}

A review of literature on fuel-saving alternatives in the trucking industry was the starting point for this project. Research reports, journal articles, and conference papers were the primary documents reviewed. Proprietary materials on specific fuel-saving equipment were also consulted. Appendix A lists the final set of sources used to develop and summarize data on fu-1-saving measures included in the matrix.

\subsection{DEVELOPMENT OF DRAFT MATRIX}

The draft matrix of truck energy efficlency measures was developed primarily by extracting and summarizing the following information from the review literature:

- Technical description.

- Energy-savings percentages claimed in demonstrations.

- Estimates of fuel-cost savings and payback periods.

- Estimates of required investment, maintenance, and other costs of implementation.

- Advantages, disadvantages, and related information. 
Estimates of cost savings for each option were calculated rather than taken directly from data sources. Two early assumptions in the project were that (1) truck operators engage in energy-saving efforts primarily to reduce costs and (2) a range of potential cost savings for various types of operations would be useful. Therefore, parametric assumptions were made for representative intracity and intercity truck operations to develop low and high diesel fuel use rates, respectively. These rates were then comblned with the estimated fuel-savings percentage for each option, factored by an estimated diesel fuel price of $\$ 1 / g a l$. This procedure allowed an estimated range of cost savings to be included in the matrix for most options. For other measures, however, neither percentage- nor a dollar-based fuel cost savings could be included in the matrix because those savings, though known to exist, vary by individual truck and route traveled. In those cases, savings were identified simply as truck-and-route-specific (TRS) so that matrix users could evaluate potential savings for their particular circumstances. The same individualized situations apply to financial payback periods for many options, and the TRS entry was also made in those cases.

Another Important assumption made for each fuel-saving measure was the Identification of its relevant market sector(s). This was done so that matrix users could focus more easily on alternatives applicable to their types of vehicles and operations.

Options were organized in the matrix under the following categories:

- Operations.

- Equipment purchasing and modifying.

- Fuel and lubricant changes.

- Technologies under development.

\subsection{INDUSTRY COORDINATION MEETING AND REVISION OF MATRIX}

An all-day industry coordination meeting was held on August 26, 1982, in Willowbrook, Illinols (a southwestern suburb of Chicago). A broad crosssection of trucking industry expertise was represented. The participants (1isted in Appendix B) Included three common-carrier truck operators, three private truck fleet operators, and representatives of the American Trucking Associations, the Canadian TRUCKSAVE Program, and a trucking trade journal, as well as two trucking consultants (one of whom was formerly sentor general engineer of the U.S. Voluntary Truck and Bus Fuel Economy Program in the U.S. Department of Transportation) and two ANL program managers. Detalled discussions were held on each measure of the matrix, and all changes and other conclusions were made by consensus, sometimes after lengthy discussions. The results of this meeting are summarized in Sec. 4. 
The meeting was tape-recorded and notes were taken by ANL representatives. After the meeting, these records were carefully reviewed and used to revise the matrix, a copy of which was then sent to each participant for final comments. Section 4 contains a detalled discussion of the substantive matrix revisions that were made to incorporate the meeting results.

\subsection{PRESS RELEASE AND DISTRIBUTION OF MATRIX}

Following revision of the matrix, 1870 copies were malled to individuals and groups within the U.S. trucking industry, including operators of private truck fleets; appropriate military trucking groups and officials were also among the recipients. Approximately 100 additional copies were mailed to truck industry researchers and consultants who may be able to affect trucking activities directly or indirectly. The addressees were selected from the mailing list of the U.S. Voluntary Truck and Bus Fuel Economy Program. Another 100 coples were sent to the Canadian TRUCKSAVE Program for that group to publicize and distribute upon request. The cover letter for the copies distributed in the U.S. stated that the reference list was a source of additional information concerning fuel-saving measures and was available on request. The resulting requests provided a low-cost indication of industry interest.

In addition, a press release highlighting the truck matrix and industry coordination meeting was sent to various truck- and transportation-industry publications. The release also described the rail and maritime matrices and the results of all three industry coordination meetings. The following publications have printed the release: Road King, Fleet Maintenance and Specifying, Transport Topics, and Transportation Business.

The release (reproduced in Appendix C) included an ANL mailing address to which requests for matrix coples could be sent. This was done to distribute the matrix as widely as possible to interested truckers and other parties not included in the orfginal malling and to provide another low-cost measurement of industry response. Of particular note are the 572 requests for the matrix received through Road King, a trade journal for Independent truckers.

\subsection{REPORT PREPARATION AND DISTRIBUTION}

Preparation and distribution of this final report are the final tasks in this research. This document is intended for distribution to (1) appropriate industry organizations such as the American Trucking Association and the Private Truck Counc11, (2) government agencies such as the U.S. Departments of Energy and Transportation, and (3) truck operators who request additional information about the fuel-savings options in the matrix. 
3 DESCRIPTION OF MATRIX

The final version of the matrix developed in this project is included here as Table 1.*. The following sections describe the information found in each matrix column.

\subsection{FUEL-SAVING MEASURES}

The fuel-saving measures listed in the first column of the matrix are divided into four categories:

- Operations.

- Equipment purchasing and modifying.

- Fuel and lubricant changes.

- Technologies under development.

Many fuels-saving measures have several submeasures presented, as for example, in the operations improvement category, where more than 20 separate 1 tems are listed under six measures. The list of measures in the matrix, although comprehensive, should not be considered as all-inclusive.

\subsection{CLAIMED FUEL SAVINGS}

Fuel-savings percentages in the second column, unless indicated otherwise by footnote, are those claimed in the references cited. The word "claimed" is used in the column heading because, for many measures, claims of savings varied among sources. In addition, because each truck and operating route is different, the meeting particlpants agreed that every user of the matrix should not expect exactly the same result. Even so, for many optlons, meeting participant and reference estimates of fuel savings were close enough to warrant the use of only one number.

However, some conservation measures were considered to be truck-androute-specific. This occurred when no estimated range of savings was found or when meeting participants disagreed with the reference claims and yet conceded that some savings potential was 11kely.

*The matrix in Table 1 is identical to that distributed to the trucking industry except for minor editorial revisions in format and wording. 


\subsection{ASSUMED FUEL USE}

The third column provides a simplified parametric basis for estimating the annual diesel fuel cost savings implied by the claimed fuel-savings percentages in the second column. After discussions with meeting participants, it was assumed that a representative intracity truck would consume 4000 gallons annually $(40,000 \mathrm{ml}$ [ 10 miles per gallon [mpg]) and a representative interclty truck would consume 20,000 gallons annually $(100,000$ mi ( $5 \mathrm{mpg})$. Even though these assumptions are for diesel-powered trucks, the same assumptions should be applicable to intracity operations using gasolinepowered vehicles.

\subsection{CLAIMED 1983 FUEL COST SAVINGS}

The savings in this column were derived by multiplying the claimed fuel-savings percentages in the second column by an estimated diesel fuel cost of $\$ 1 / \mathrm{gal}$.

The cost savings estimated in this column are gross, rather than net. They do not take into account (1) the allocations of investment costs necessary to realize the savings or (2) the higher maintenance or other operating costs required by some fuel-saving options (e.g., use of larger trailers). Nevertheless, they do translate energy savings into gross dollar savings, which relate more closely to profit, the primary business motive.

\subsection{ADDITIONAL 1983 COSTS}

Three columns are included under this heading: investment, maintenance, and other. Information was difficult to obtain for these columns and there are many blank spaces. This lack of information resulted mostly from a primary emphasis in the literature on the energy and cost savings of conservation measures rather than on added costs. In addition, the additional costs varied so widely in some cases that a "TRS" entry was used in the matrix to cover the wide range. Nevertheless, matrix users should realize that added costs do accompany many of the measures and must be determined for individual trucks in order to calculate the payback periods on which investment decisions are usually based.

\subsection{APPROXIMATE PAYBACK PERIOD}

The payback periods in this column are labeled as approximate because they are based on claimed fuel savings, which often vary by truck and route. Similarly, the investment, maintenance, and other costs required by fuelsavings measures also vary by truck and sometimes by route as well. Nevertheless, the payback periods listed, espectally when less than one or two years, should provide truck operators with useful deciston-making information. 
Payback period calculations were not attempted for the matrix primarily because even when annual fuel savings could be estimated, there were insufflcient data on additional costs assoclated with the conservation measures. In addition, such calculations would be unwieldy and Inappropriate for a summary matrix because reviews of relevant payback analyses revealed the need for highly individualized (vehicle-specific) and detalled data, in addition to projections of a wide range of petroleum price escalations and/or reductions.

\subsection{ASSUMED MARKET SECTORS}

Many of the energy conservation measures are applicable to all trucks, but others are designed for certaln truck types (e.g., tractor-trailers, plckup-and-delivery vans), ages, sizes, (e.g., twin traflers), speeds, or engine types (e.g., diesel, gasoline). This column was added to help truck operators focus more easily on the appropriate measures for their vehicles.

\subsection{REMARRS}

Important information concerning operator experiences and the functlons, advantages, disadvantages, and risks of the fuel-saving optlons is presented in the final column. The column provides qualitative, and in some cases quantitative, information vital to proper evaluation of the measures. 
Table 1 Matrix of Truck Energy Efficiency Measures

\begin{tabular}{|c|c|c|c|c|c|c|c|c|c|}
\hline \multirow[b]{2}{*}{ Puel-Sevinge Measure } & \multicolumn{6}{|c|}{ Per Truck } & \multirow[b]{2}{*}{$\begin{array}{c}\text { Approx. } \\
\text { Payback } \\
\text { Pertod (1f } \\
\text { avallable) }\end{array}$} & \multirow[b]{2}{*}{$\begin{array}{l}\text { Ass umed } \\
\text { Market } \\
\text { Sectora }\end{array}$} & \multirow[b]{2}{*}{ Remark: } \\
\hline & $\begin{array}{c}\text { Clalmed } \\
\text { Puel } \\
\text { Savinge } \\
(x)^{2}\end{array}$ & $\begin{array}{l}\text { Assumed } \\
\text { Fuel Use } \\
\left(10^{3} \mathrm{gal} / \mathrm{yr}\right)\end{array}$ & $\begin{array}{l}\text { Clalaed } \\
\text { 1983 } \\
\text { Annual } \\
\text { Puel Cost } \\
\text { Savings } \\
\text { (\$) }\end{array}$ & \multicolumn{3}{|c|}{ Addictonal 1983 Costs (S) } & & & \\
\hline \multicolumn{10}{|l|}{ OPERATIONS } \\
\hline $\begin{array}{l}\text { 1. Driver Improvenenta: } \\
\text { Aevelopaent of driver } \\
\text { understanding, cotiva- } \\
\text { cton, cooperation, and } \\
\text { participation (prefer- } \\
\text { ably through mage- } \\
\text { ment progran) (Refs. } \\
1-6,61,82-86)\end{array}$ & --- See & Measure is be & low --.-- & $\begin{array}{l}\mathbf{0} \\
\mathbf{0}\end{array}$ & $\begin{array}{l}0 \\
0\end{array}$ & $\begin{array}{l}\text { TRS } \\
\text { TRS }\end{array}$ & $\begin{array}{l}<6 \text { months } e \\
<6 \text { months }\end{array}$ & $\begin{array}{l}\text { All trucks } \\
\text { Al ? trucks }\end{array}$ & 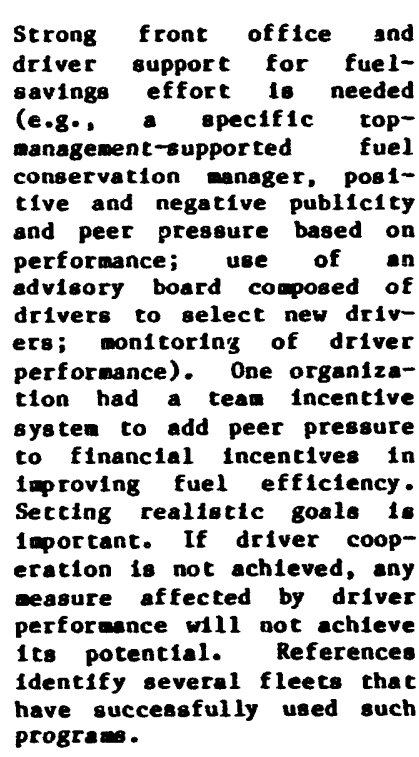 \\
\hline $\begin{array}{l}\text { b. Improve driving } \\
\text { practices }\end{array}$ & $\begin{array}{l}\text { Up tu } 12^{e} \\
\text { (total) }\end{array}$ & $\begin{array}{r}4 \\
20\end{array}$ & $\begin{array}{l}\text { Up to } 480 \\
\text { Up to } 2400 \\
\text { (totals) }\end{array}$ & ב-ב See & $\begin{array}{l}\text { Remarks } \\
\text { Renarks }\end{array}$ & - & $\begin{array}{l}\text { See Remarks } \\
\text { See Remarks }\end{array}$ & $\begin{array}{l}\text { All cruck: } \\
\text { A11 trucks }\end{array}$ & $\begin{array}{l}\text { Combined avings (only) of } \\
\text { lb(1) through ib( }(7) \text { below. } \\
\text { of fsetting equipment costs } \\
\text { are Identified In the } \\
\text { Investment column. These } \\
\text { savings are aleo of foet } \\
\text { somewhat by the costs of } \\
\text { driver training, thich are } \\
\text { Indicated as truck-and- } \\
\text { route-specific in the } \\
\text { "other" additional cost } \\
\text { colum for la above. }\end{array}$ \\
\hline
\end{tabular}


Table 1 (Cont'd)

Per Truck

\begin{tabular}{|c|c|c|c|c|c|c|c|c|c|}
\hline \multirow[b]{2}{*}{ Puel-Sevinge Measure } & \multicolumn{6}{|c|}{ Per Truck } & \multirow[b]{2}{*}{$\begin{array}{l}\text { Approx. } \\
\text { Payback } \\
\text { Period (1f } \\
\text { avallable) }\end{array}$} & & \multirow[b]{2}{*}{ Remarks } \\
\hline & $\begin{array}{l}\text { Clalued } \\
\text { Puel } \\
\text { Savinge } \\
(x)\end{array}$ & 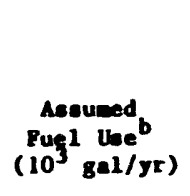 & $\begin{array}{l}\text { Clat ined } \\
1983 \\
\text { Annual } \\
\text { Puel Cost } \\
\text { Savingec } \\
\text { ( } \$ \text { ) }\end{array}$ & $\frac{\text { Additional }}{\text { Inveatent }}$ & 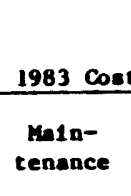 & other & & $\begin{array}{l}\text { Aseuned } \\
\text { Market } \\
\text { Sectors }\end{array}$ & \\
\hline OPERATIONS (Cont'd) & & & & & & & & & \\
\hline $\begin{array}{l}\text { (1) Elininate un- } \\
\text { necessary 1d11ng } \\
\text { (Refs. 1-7, 62, 63, } \\
\text { 82-86). }\end{array}$ & - & See Measure 1 & $1 b$ - & $\begin{array}{c}\text { See } \\
\text { Remarke }\end{array}$ & o & TRS & TRS & All trucke & 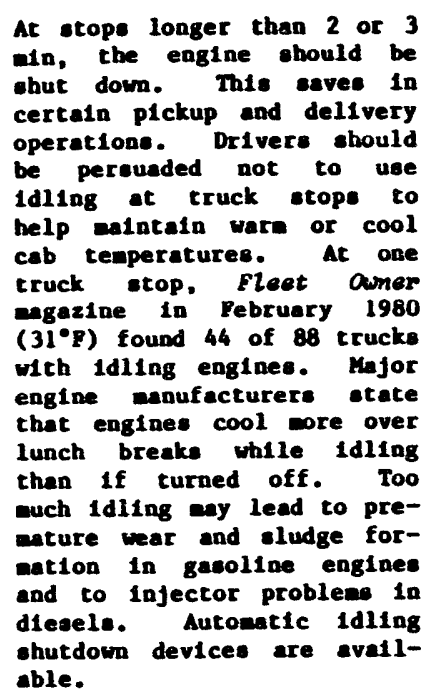 \\
\hline $\begin{array}{l}\text { (2) Never exceed } 55 \\
\text { oph opeed l1mit or } \\
\text { ottenpt to defeat } \\
\text { purpose of governor } \\
\text { equipment (Refe. } \\
1-10,64,65,82-86 \text { ) }\end{array}$ & $\begin{array}{l}1.5-2.0 \\
\text { for each } \\
\text { aph re- } \\
\text { duced } \\
\text { toward } \\
55 \text { mph }\end{array}$ & 20 & TRS & $\mathbf{0}$ & 0 & TuS & $\mathrm{N} / \mathrm{A}^{\mathrm{E}}$ & All trucks & 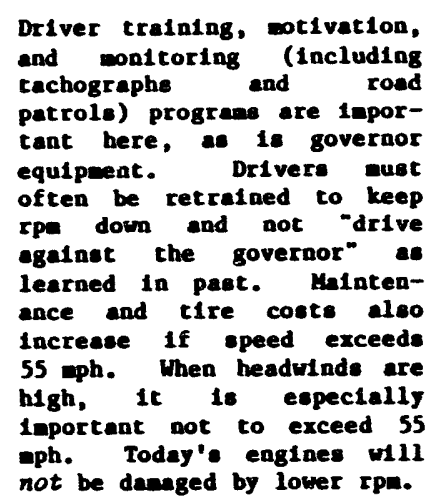 \\
\hline
\end{tabular}


Table 1 (Cont'd)

Per Truck

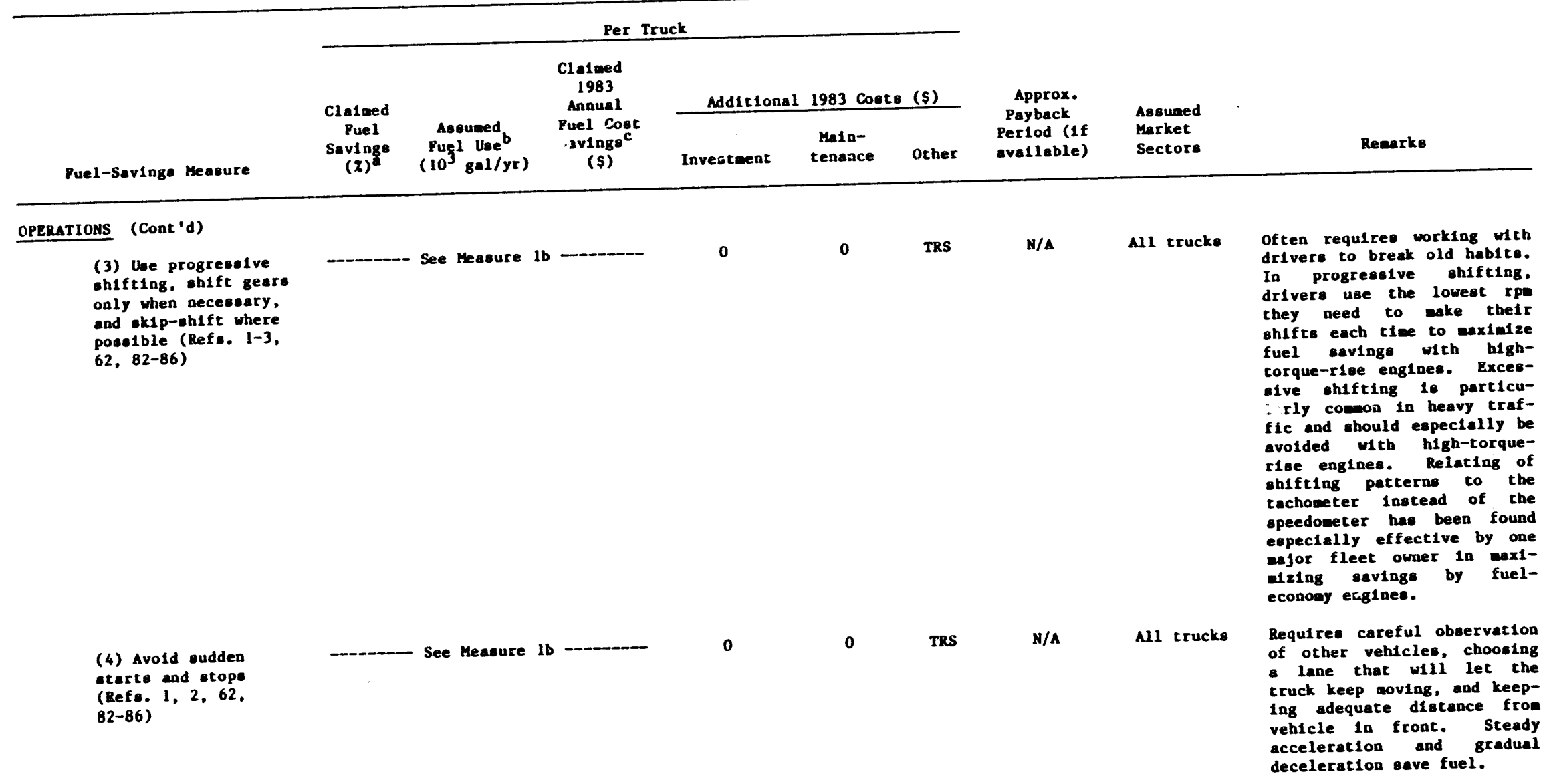


Table I (Cont'd)

\begin{tabular}{|c|c|c|c|c|c|c|c|c|c|}
\hline \multirow[b]{2}{*}{ Puel-Suv1ngs Measure } & \multicolumn{6}{|c|}{ Per Truck } & \multirow[b]{2}{*}{$\begin{array}{l}\text { Approx. } \\
\text { Payback } \\
\text { Period (1f } \\
\text { avallable) }\end{array}$} & \multirow[b]{2}{*}{$\begin{array}{l}\text { Assumed } \\
\text { Market } \\
\text { Sectors }\end{array}$} & \multirow[b]{2}{*}{ Reanks } \\
\hline & $\begin{array}{l}\text { Clained } \\
\text { Puel } \\
\text { Sevinge } \\
(x)^{3}\end{array}$ & 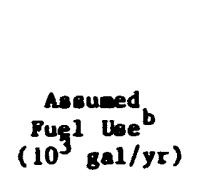 & $\begin{array}{c}\text { Clained } \\
\text { 1983 } \\
\text { Annual } \\
\text { Puel Cost } \\
\text { Savingec } \\
\text { (\$) }\end{array}$ & $\begin{array}{l}\text { Additional } \\
\text { Investment }\end{array}$ & \multicolumn{2}{|c|}{1983 Costs (\$) } & & & \\
\hline OPERATIONS (COnt'd) & & & & & & & & & \\
\hline $\begin{array}{l}\text { (5) Maintain constant } \\
\text { opeed (Refs. 1, 2, } \\
82-86 \text { ) }\end{array}$ & - & See Measure 1 & $1 b-\infty$ & 0 & 0 & TRS & $\mathbf{N} / \mathbf{A}$ & All trucks & 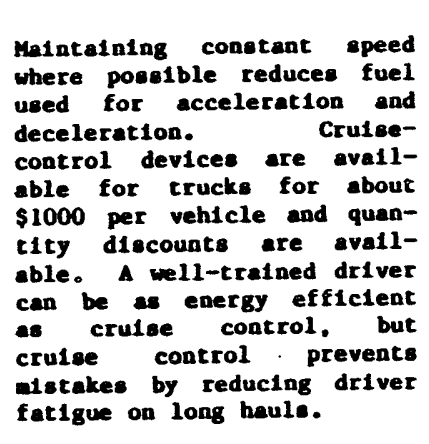 \\
\hline $\begin{array}{l}\text { (6) Monitior fuel } \\
\text { consumption (Bef. 2) }\end{array}$ & - & See Measure 1 & $1 \mathrm{~b}-\cdots$ & $\begin{array}{c}1000 \\
\text { (electronic } \\
\text { oonitoring } \\
\text { equipment) }\end{array}$ & 0 & TRS & $\mathbf{M} / \mathbf{A}$ & All trucks & $\begin{array}{l}\text { Electronic lastruments are } \\
\text { ovailable with readout } \\
\text { devices to provide data to } \\
\text { driver on fuel use during } \\
\text { trips. These also provide } \\
\text { guidence for tune-up and } \\
\text { other aintenance ocheduling } \\
\text { and are good driver-training } \\
\text { devices even where there is } \\
\text { not enough capital to equip } \\
\text { an entire fleet. }\end{array}$ \\
\hline $\begin{array}{l}\text { (7) Supervise outalde } \\
\text { fuel1ng to avold } \\
\text { "overf111." (Ref. 1) }\end{array}$ & $\cdots$ & $S_{t}:$ Measure 1 & $1 b-\cdots$ & 0 & 0 & TRS & Imeed Iate $e^{e}$ & All trucks & $\begin{array}{l}\text { Involves training and } \\
\text { monitoring of personnel who } \\
\text { f111 tanks. Also reduces } \\
\text { daange to parking areas and } \\
\text { reduces fire hazards. }\end{array}$ \\
\hline 2. Malntenance Improvement: & $\begin{array}{c}1-8 \\
\text { (total) }\end{array}$ & $\begin{array}{r}4 \\
20\end{array}$ & $\begin{array}{c}40-320 \\
200-1600 \\
\text { (totals) }\end{array}$ & -.- - See & Renark: & $\ldots$ & $\begin{array}{c}\text { See } \\
\text { Renarks }\end{array}$ & $\begin{array}{l}\text { All trucks } \\
\text { All trucks }\end{array}$ & 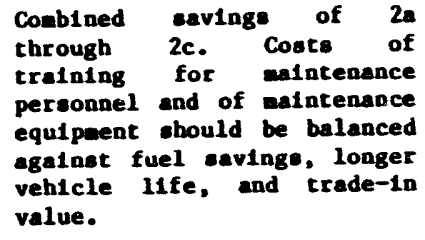 \\
\hline
\end{tabular}


Table 1 (Cont'd)

Per Truck

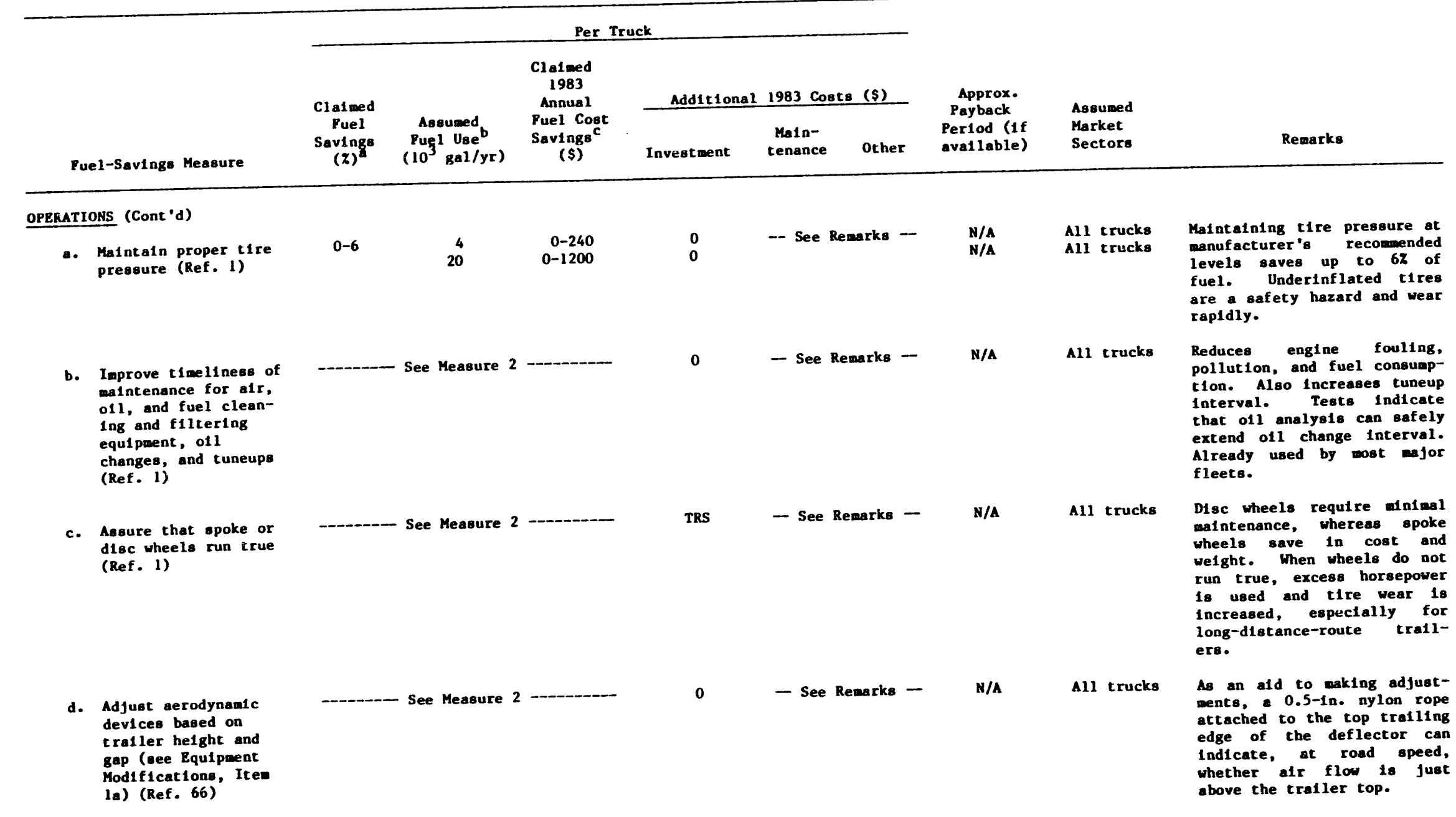


Table 1 (Cont'd)

Per Truck

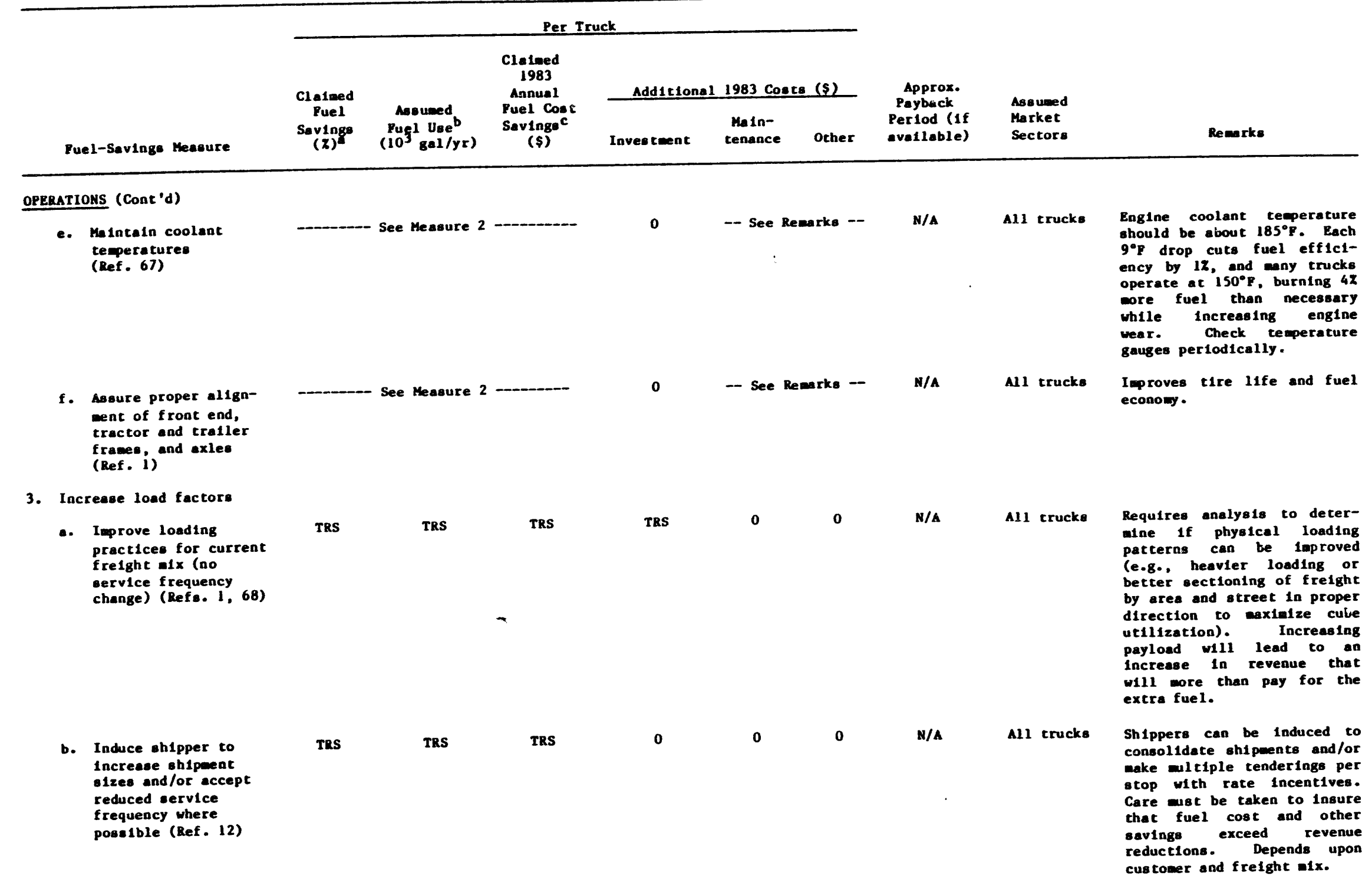


Table 1 (Cont'd)

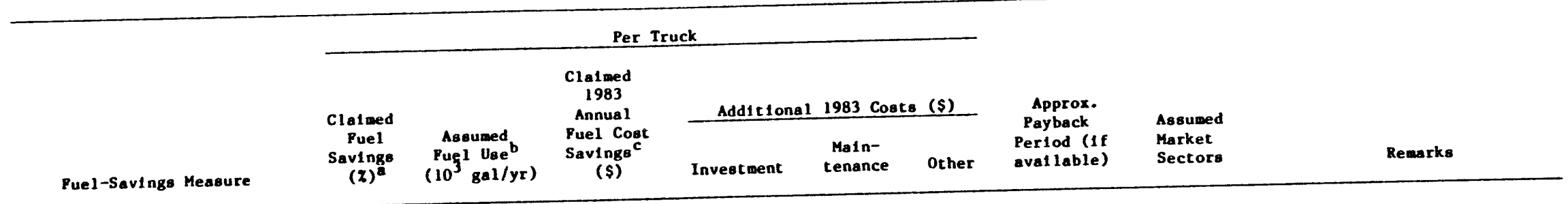

\section{OPERATIONS (Cont'd)}

4. Une of satellite, con-

bolidation, or break-bulk

a Use several satellite terminals in large cittes

TRS

TRS

TRS

TRS

0

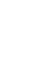

b. Use single consolidat ton terminal for mall-city operations (Ref. 13)

c. Use regional breakbulk terminals

TRS

TRS

TRS

20

TRS

TRS

TRS

0

TRS

All trucks

A11

intercity

5. Reduction of empty backhauls

a. Increase inter-

corporate backhauls
(Ref. 1)

TRS

TRS

TRS 
Table 1 (Cont'd)

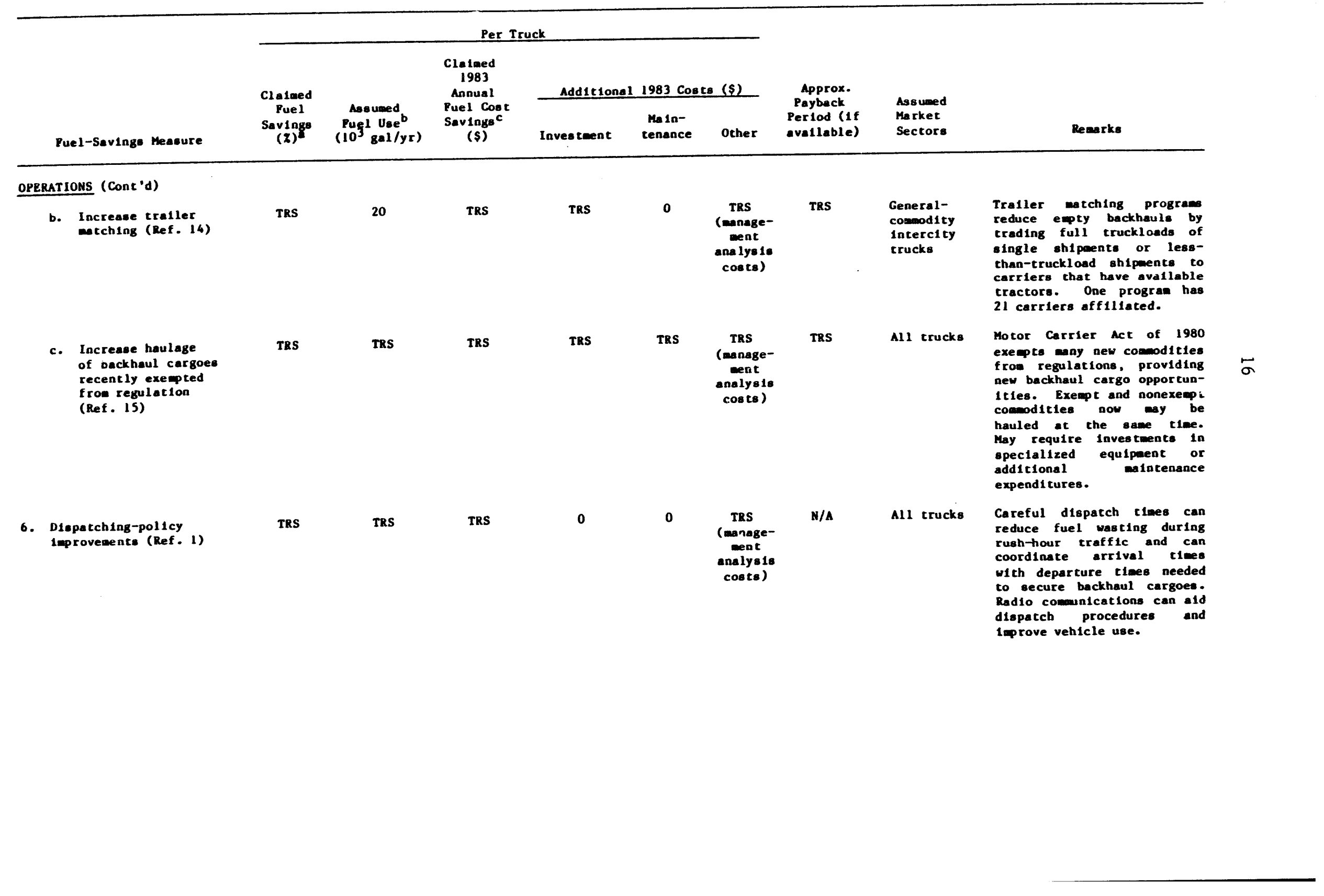


Table 1 (Cont'd)

Per Truck

Clained

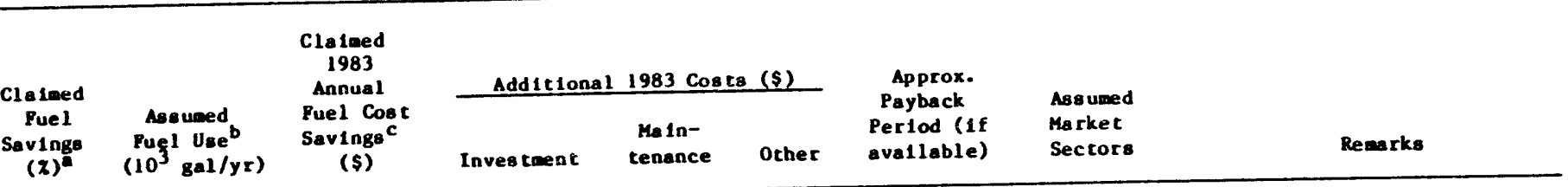

Puel-Savings Measure

Savinge Pugl Use
$(x)^{a}$$\quad\left(10^{3} \mathrm{gal} / \mathrm{yr}\right)$

Investment

cenance

avallable)

Sectors

EQUI PMENT PURCHASING

\section{AND HODIFYINC}

1. Use of resistance-reducing equil peent

a. Install serodynanic devices (e.g.., wind deflector atop

cractor cab, bubble

on traller) and use

round-cornered)

tractors and trallers
(Ref $1-7,82,87-89)$

$\begin{array}{cclccc}4 & 160-320 & 350-600 & 0 & 0 & 1 \text { year } \\ 20 & 800-1600 & \begin{array}{l}350-600 \\ \text { (aero- } \\ \text { dynamic } \\ \text { devices) }\end{array} & 0 & 0 & 6 \text { months }\end{array}$

All trucks

All trucks

devices
As truck speed Increases. aerodynamic drag increases much more quickly than reduce aerodynamic drag and are just as useful on stralght trucks as on traight trucks as on clally good for operations with light-density loads. Resulcs will be a function of vehicle configuration (tractor, traller, and gap between them). A rig with a large gap will not benefit fros present devices. Ne ther will low-speed local operators or tanker, fletbed, or bobtall operations. Tractors with high trallerless atles should use folddown deflectors. Voluntary Truck and Bus Progran participants average $6 \%$ fuel gavings. Keep trailer as low as possible. 
Table 1 (Cont'd)

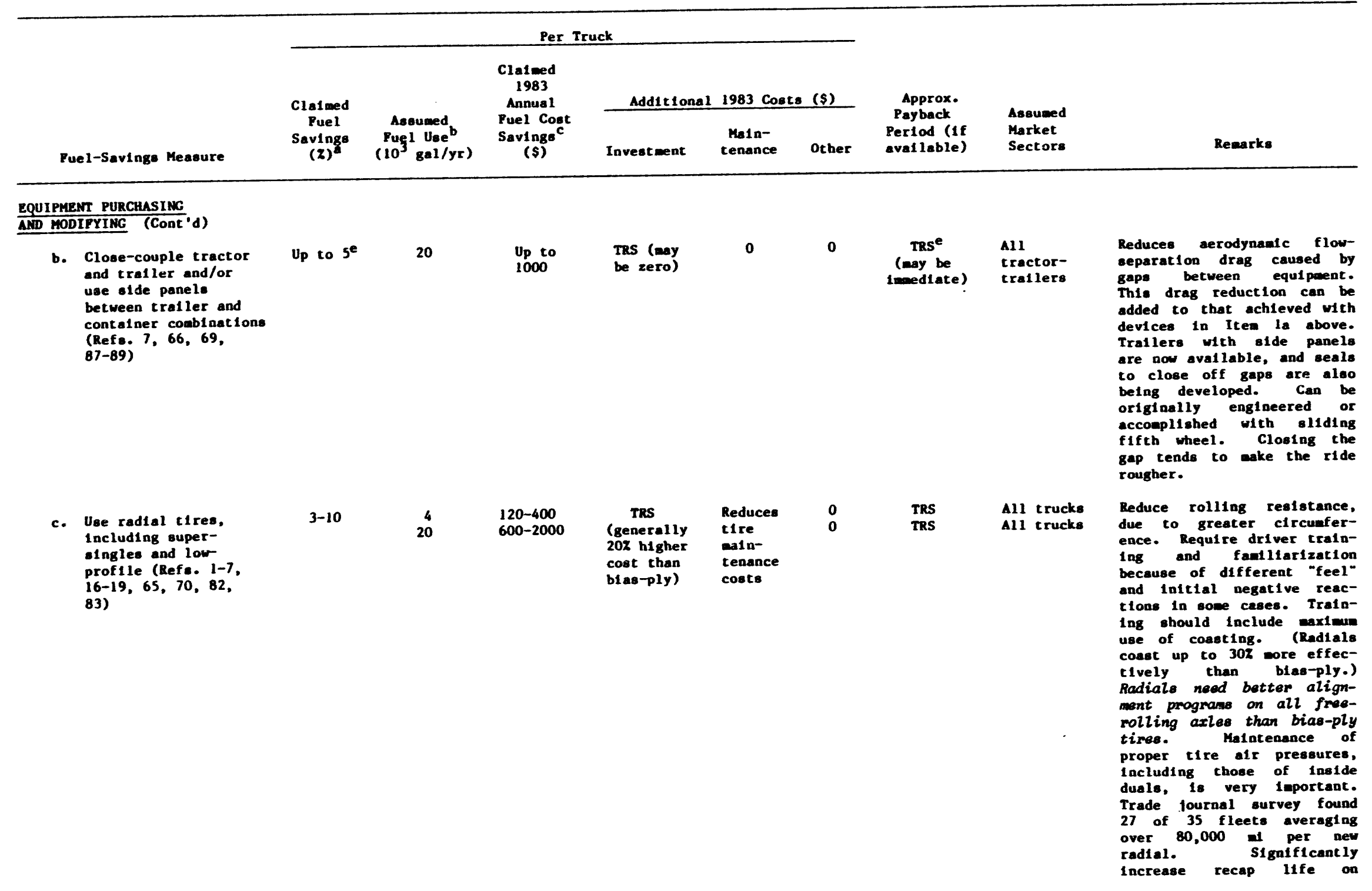


Table 1 (Cont'd)

Per Truck

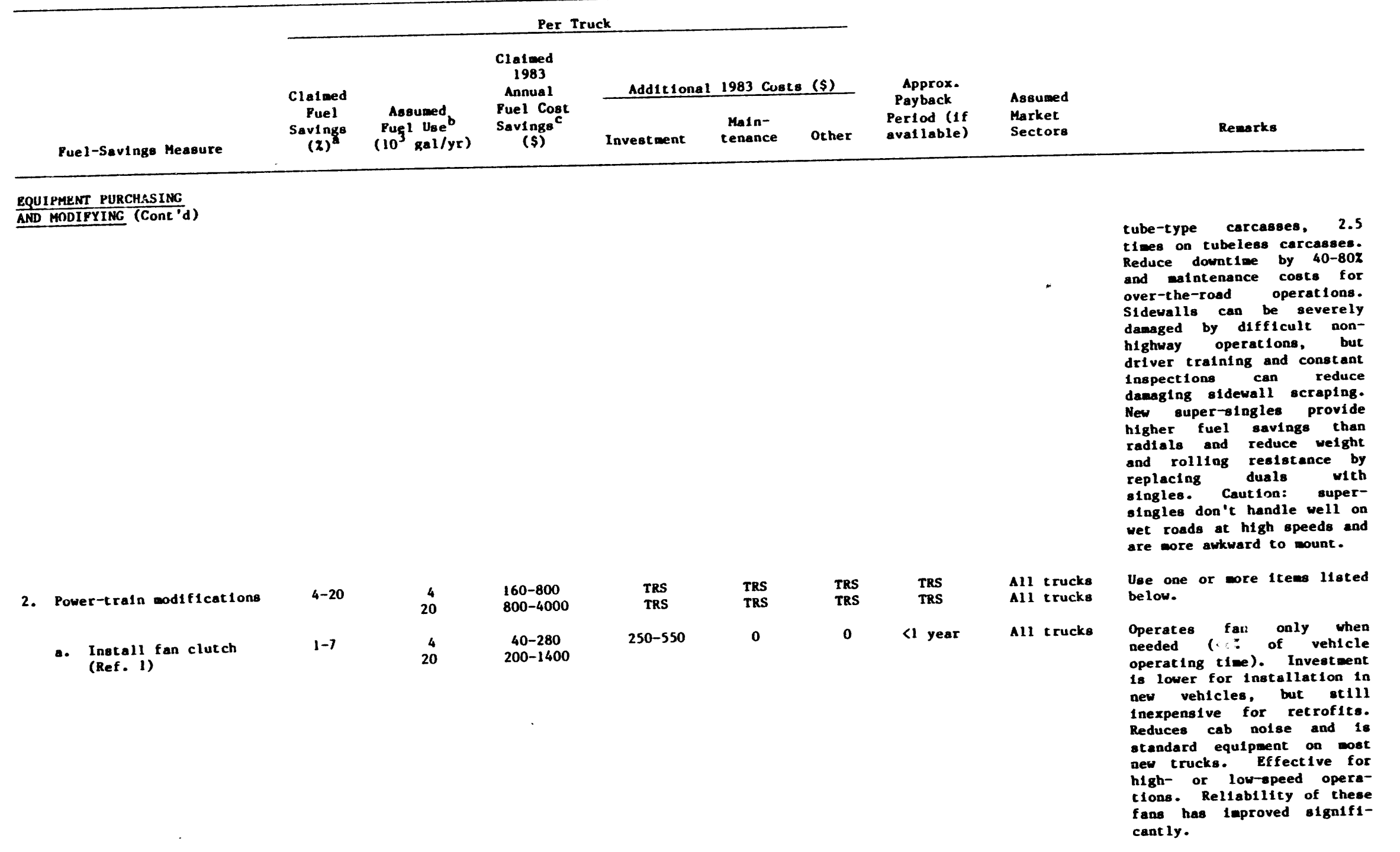


Table 1 (Cont'd)

Per Truck

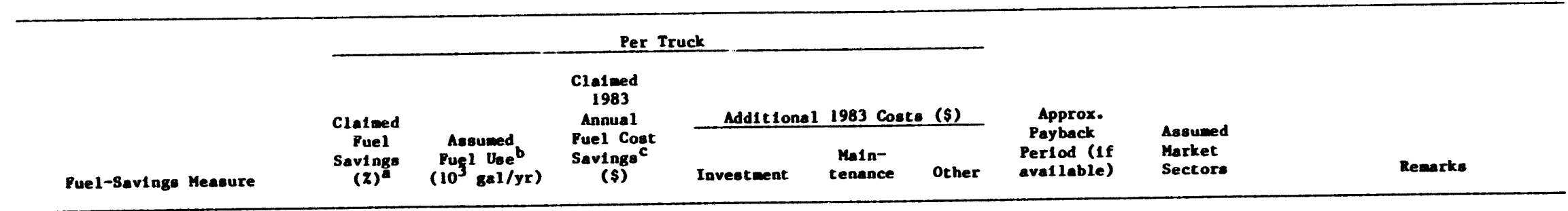

QUIPMRNT PURCHASIMC

AND MODIFYING (Cont'd)

b. Inetall and/or adjust governors for maximu: fuel efficiency (Ref $s$. $1,7)$

(1) Adjust engine-rpa governor for maxi mum fficlency (Refs. 1 , $7,69)$
TRS

TRS

TRS

TRS governor for maximun

$1,7,69,71$ )
All trucks

Inacallation establishes aximen engine speed, thus reducting fuel we. example, governed engibe peed can be reduced by 200 300, while the truck stil has a maximin speed of 60 saves considerabl fuel.

Lints rand speed below the geared road speed. Hore expensive and complex than engl ne governor, as well as making the truck harder to drive and requitring more shifting, but allows effic1ent engine operation. Conputer simulat ion indicated 5X apg Inprovement. 
Table 1 (Cont'd)

Per Truck

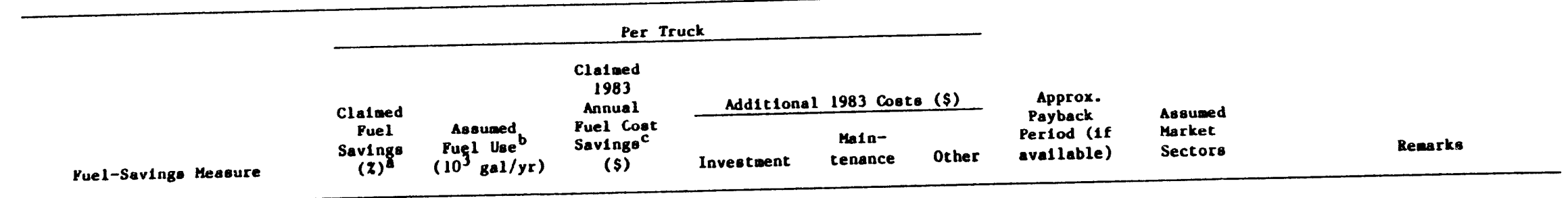

EUIPMENT PURCHASING

AND MODIFYING (Cont'd

c. Install lowest rear axle ratio appropri ate for desired

(Ref.. 1, 1, 19, 65)

4
20

Up to 200

0
0
(new
ractors)

$\mathbf{0}$

TRS (ractors)

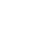

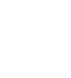

Install transutesion

TRS

TRS

TRS

TRS

TRS

TRS

TRS

with fewer fixed gear

e. Subetitute dlesel for gasoline engine

(1) Large or mediua
trucks (Ref. 19)
Up to 40

4
20
Up to 1600

Up to 8000
3000
3000
All trucks
All trucks

Priearily for new tractor purchases because retiufit as 3.55 to 3.7 are belag used for over-the-roed tractors. Caut1on: Truck must have a trangnission and rear axle combination to meet gteepest-grade starting conditions for the particular operations. Also, trucks with low-rpa cruise capabil1ty of 55 mp are still capable of reaching quite high, fuel-1nefficient speeds. Th1s can be controlled with a road-rpeed governor.

All rrucks

Pewer gears to shift enable better driver training and control. Cear friction 1s also reduced. Overdrive 1s applications.

New, long- Best application 10 for distance large, long-distance trucks. trucks Operations that have traded up from basll to cediub stantial savings. Engine iffe is 100-200Z longer for diesels. Need 15,000-20,000 -1les to reach payoff for diesel vs. gasoline. 
Table 1 (Cont'd)

\begin{tabular}{|c|c|c|c|c|c|c|c|c|c|}
\hline \multirow[b]{2}{*}{ Puel-Savinge Heasure } & \multicolumn{6}{|c|}{ Per Truck } & \multirow[b]{2}{*}{$\begin{array}{l}\text { Approx. } \\
\text { Payback } \\
\text { Period (1f } \\
\text { avalloble) }\end{array}$} & \multirow[b]{2}{*}{$\begin{array}{l}\text { Assumed } \\
\text { Market } \\
\text { Sectora }\end{array}$} & \multirow[b]{2}{*}{ Rewark: } \\
\hline & $\begin{array}{c}\text { Clained } \\
\text { Puel } \\
\text { Savinge } \\
(x)^{\mathbf{Z}}\end{array}$ & $\begin{array}{l}\text { Asaused } \\
\text { Puesl Use } \\
\left(10^{3} \mathrm{gal} / \mathrm{yr}\right)\end{array}$ & $\begin{array}{c}\text { Claimed } \\
1983 \\
\text { Annual } \\
\text { Puel Cogt } \\
\text { Savingec } \\
\text { ( } \$ \text { ) }\end{array}$ & Additional & $1983 \mathrm{Cost}$ & $\frac{(\$)}{\text { Other }}$ & & & \\
\hline $\begin{array}{l}\text { EQUIPMENT PURCHASING } \\
\text { MID HODIFYING (Cont' }\end{array}$ & & & & & & \multirow[b]{2}{*}{$\begin{array}{c}40 x \\
\text { decrease } \\
\text { to } 90 x \\
\text { increase } \\
\text { In oper- } \\
\text { ating } \\
\text { costs }\end{array}$} & \multirow[b]{2}{*}{ TRS } & \multirow[b]{2}{*}{$\begin{array}{l}\text { New, } \\
\text { short-, } \\
\text { mediun- } \\
\text { distance } \\
\text { trucks }\end{array}$} & \\
\hline $\begin{array}{l}\text { (2) Sen11 trucks } \\
\text { (Refs. 19-23) }\end{array}$ & $20-50$ & 4 & $800-2000$ & 2000 & $\begin{array}{c}\text { See } \\
\text { Reaarks }\end{array}$ & & & & 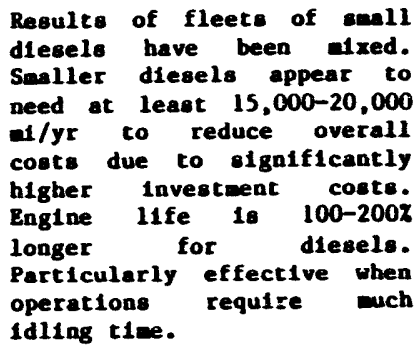 \\
\hline $\begin{array}{l}\text { f. Use tag (dead) axle } \\
\text { Refs. } 1,7,19,24)\end{array}$ & $2-5$ & $\begin{array}{r}4 \\
20\end{array}$ & $\begin{array}{l}80-200 \\
400-1000\end{array}$ & $\begin{array}{l}\mathbf{0} \\
\mathbf{0}\end{array}$ & $\begin{array}{l}\mathbf{0} \\
\mathbf{0}\end{array}$ & $\begin{array}{l}\mathbf{0} \\
\mathbf{0}\end{array}$ & $\begin{array}{l}\text { TRS } \\
\text { TRS }\end{array}$ & $\begin{array}{c}\text { See } \\
\text { Remarks }\end{array}$ & 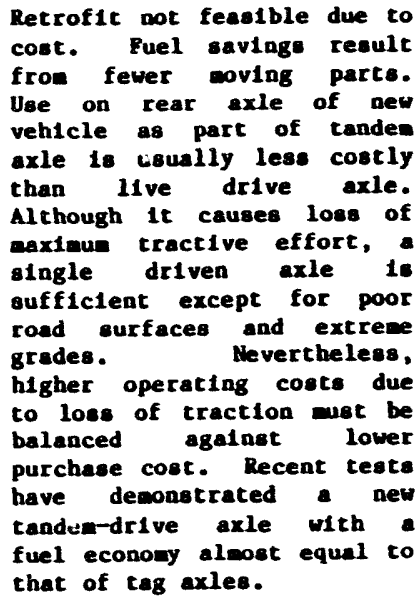 \\
\hline
\end{tabular}


Table 1 (Cont'd)

Per Truck

\begin{tabular}{|c|c|c|c|c|c|c|c|c|c|c|}
\hline \multirow{2}{*}{\multicolumn{2}{|c|}{ Puel-Savinge Meacure }} & \multicolumn{6}{|c|}{ Per Truck } & \multirow[b]{2}{*}{$\begin{array}{l}\text { Approx. } \\
\text { Payback } \\
\text { Perlod (1f } \\
\text { aval lable) }\end{array}$} & \multirow{2}{*}{$\begin{array}{l} \\
\text { Assumed } \\
\text { Market } \\
\text { Sectors }\end{array}$} & \multirow[b]{2}{*}{ Remarks } \\
\hline & & $\begin{array}{l}\text { Clained } \\
\text { Puel } \\
\text { Savings } \\
(x)^{8}\end{array}$ & $\begin{array}{l}\text { Assuned } \\
\text { Puefl Use } \\
\left(10^{\mathrm{b}} \mathrm{ga1} / \mathrm{yr}\right)\end{array}$ & $\begin{array}{l}\text { Claised } \\
1983 \\
\text { Annual } \\
\text { Puel Cost } \\
\text { Savingsc } \\
\text { (\$) }\end{array}$ & $\begin{array}{l}\text { Additional } \\
\text { Investment }\end{array}$ & $\frac{1983 \text { Costs }}{\substack{\text { Main- } \\
\text { tenance }}}$ & other & & & \\
\hline$\frac{E Q U}{A N D}$ & $\begin{array}{l}\text { IPHENT PURCHASING } \\
\text { MODIPYING (Cont'd) }\end{array}$ & & & & & & & & & \\
\hline & $\begin{array}{l}\text { g. Use higii-torque, } \\
\text { low-rpa diesel } \\
\text { engine (Refs. 1, 25, } \\
\text { 26) }\end{array}$ & $5-15^{d}$ & $\begin{array}{r}4 \\
20\end{array}$ & $\begin{array}{c}200-600^{8} \\
1000-30008\end{array}$ & $\begin{array}{l}500-600^{8} \\
500-600^{8}\end{array}$ & $\begin{array}{l}\mathbf{0} \\
\mathbf{0}\end{array}$ & $\begin{array}{l}0 \\
0\end{array}$ & $\begin{array}{l}\text { TRS } \\
\text { TRS }\end{array}$ & $\begin{array}{l}\text { All new } \\
\text { trucks }\end{array}$ & $\begin{array}{l}\text { These engines are direct- } \\
\text { lnjeciton, turbocharged } \\
\text { diesels with high torque at } \\
\text { low engine ppeeds ( } 1600 \text { rpa } \\
\text { and lower) and low governed } \\
\text { englne speeds. Hoise } 18 \\
\text { reduced. Manufacturers have } \\
\text { program to educate owners } \\
\text { and drivers for axinization } \\
\text { of fuel savings from these } \\
\text { engines. }\end{array}$ \\
\hline & $\begin{array}{l}\text { h. Use diesel turbo- } \\
\text { charged/aftercooled } \\
\text { eagine (Ref. 65) }\end{array}$ & TRS & TRS & TRS & TRS & TRS & TRS & TRS & $\begin{array}{l}\text { All new } \\
\text { trucks }\end{array}$ & $\begin{array}{l}\text { The aftercooler lowers } \\
\text { intake temperature, which in } \\
\text { turn reduces peak cyllinder } \\
\text { and exhaust teaperature and } \\
\text { reduces compression work } \\
\text { requirements. }\end{array}$ \\
\hline 3. & $\begin{array}{l}\text { Install mon1toring } \\
\text { equi pment } \\
\text { A. Tachoseter (Ref. 3) }\end{array}$ & TRS & TRS & TRS & $\begin{array}{l}130 \\
\text { (Frequently } \\
\text { otandard } \\
\text { equipaent) }\end{array}$ & $\mathbf{0}$ & TRS & $<1$ year $^{e}$ & All trucks & $\begin{array}{l}\text { Training drivers to tie } \\
\text { ohifting patterns to tach- } \\
\text { ometer instead of speed- } \\
\text { ometer is iaportant to the } \\
\text { effective use of fuel- } \\
\text { economy engines. }\end{array}$ \\
\hline
\end{tabular}


Table 1 (Cont'd)

\begin{tabular}{|c|c|c|c|c|c|c|c|c|c|c|c|}
\hline \multirow{3}{*}{\multicolumn{2}{|c|}{ Puel-Savings Measure }} & \multicolumn{6}{|c|}{ Per Truck } & \multirow{3}{*}{$\begin{array}{l}\text { Approx. } \\
\text { Peyback } \\
\text { Period (1f } \\
\text { avallable) }\end{array}$} & \multirow{3}{*}{$\begin{array}{l}\text { Assumed } \\
\text { Market } \\
\text { Sectors }\end{array}$} & \multirow[b]{3}{*}{ Rewark• } & \\
\hline & & \multirow{2}{*}{$\begin{array}{c}\text { Clalued } \\
\text { Puel } \\
\text { Savinge } \\
(x)^{8}\end{array}$} & \multirow{2}{*}{$\begin{array}{l}\text { Aasumed } \\
\text { Pues1 Uae b } \\
\left(10^{3} \mathrm{gel} / \mathrm{yr}\right)\end{array}$} & \multirow{2}{*}{$\begin{array}{c}\text { Cla1sed } \\
\text { 1983 } \\
\text { Annual } \\
\text { Puel Coget } \\
\text { Sevinge } \\
\text { (\$) }\end{array}$} & \multicolumn{3}{|c|}{ Additional 1983 Coste (\$) } & & & & \\
\hline & & & & & Investment & tenance & other & & & & \\
\hline \multicolumn{12}{|c|}{$\begin{array}{l}\text { EQUI PRENT PURCHASIMG } \\
\text { AND HODIFYING (Cont' }\end{array}$} \\
\hline & $\begin{array}{l}\text { D. Tachograph } \\
\text { (Refs. 1-6, 10) }\end{array}$ & TRS & TRS & TRS & $\begin{array}{c}290-370 \\
\text { (mechanical) } \\
670 \\
\text { (electronic) }\end{array}$ & 0 & $\begin{array}{c}\text { TRS } \\
\text { (manage- } \\
\text { ment } \\
\text { analysis } \\
\text { coots) }\end{array}$ & $<1$ year $^{e}$ & All trucks & 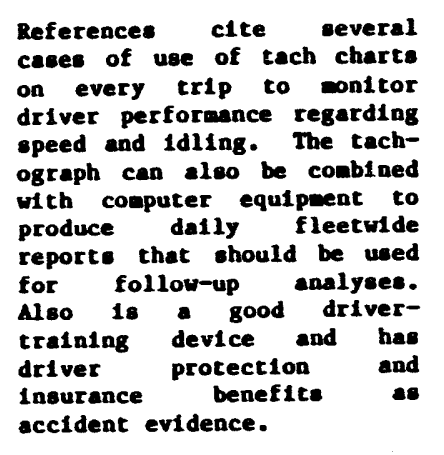 & $\underset{E}{N}$ \\
\hline 4. & 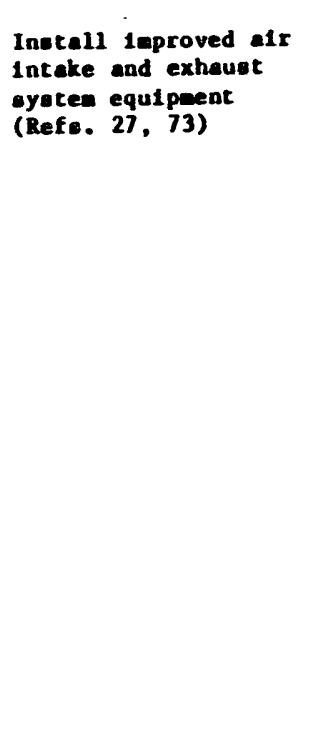 & $\begin{array}{l}\text { Up to } \\
2.5^{e}\end{array}$ & 20 & $\begin{array}{l}\text { Up to } 100 \\
\text { Up to } 500\end{array}$ & $\begin{array}{c}\text { 5-13 } \\
\text { (alr f1lter } \\
\text { Indicators) }\end{array}$ & 0 & 0 & TRS & $\begin{array}{l}\text { All diesel } \\
\text { trucks }\end{array}$ & 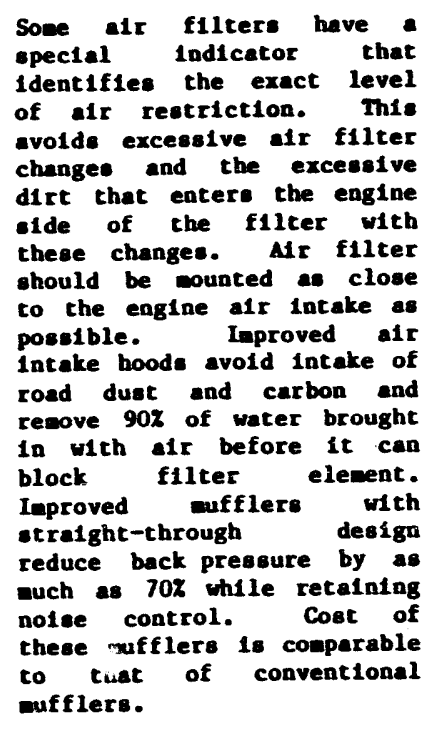 & \\
\hline
\end{tabular}


Table 1 (Cont'd)

Per Truck

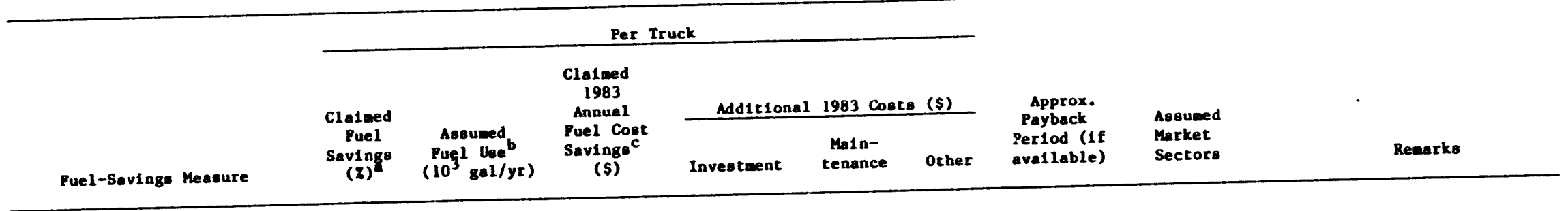

EQUI PMENT PURCHASING

AND HODIPYING (Cont'd)

5. Hase of larger or mitiple traller.

A. Use 45-ft trallers Inotead of 40-ft trailer

(Refe. 28, 29, 74)

b. Use twin 27-ft trallers insteed of single $40-\mathrm{ft}$

Refe. 28, 29)

c. Uae triple 27-ft trailers Instead
of single $45-\mathrm{ft}$ of sing

(Refs. 28-32)

d. Use triple 27-ft trallers Inates of twin 27

(Refs. 33, 34)

e. Use longer trucks, or drop trallers, wap bodies, or dron Refs. 1, 35)

Up to 10
(per ton-
nle)

Up to 22

(per ton-

ale)

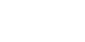

Up to 15
(per ton-

(per )

20

Up to 3000

$$
\begin{gathered}
21,000- \\
25,000 \\
\text { (1ncludes } \\
\text { two } \\
\text { doll1es) }
\end{gathered}
$$

Up to 27
(per ton-
(1le)

20

Up to 5400

15,000

TRS

TRS

IRS

o

(1ncludes

ingle-axi

dolly) 
Table 1 (Cont'd)

\begin{tabular}{|c|c|c|c|c|c|c|c|c|c|c|}
\hline \multirow{3}{*}{\multicolumn{2}{|c|}{ Puel-Sav1age Measure }} & \multicolumn{6}{|c|}{ Per Truck } & & & \\
\hline & & \multirow{2}{*}{$\begin{array}{l}\text { C2elaned } \\
\text { Puel } \\
\text { Sevinge } \\
(z)^{2}\end{array}$} & \multirow{2}{*}{$\begin{array}{l}\text { Assumed } \\
\text { Puspl Use } \\
\left(10^{b} \mathrm{gel}^{\mathrm{b}} / \mathrm{yr}\right)\end{array}$} & \multirow{2}{*}{$\begin{array}{l}\text { Clatwed } \\
1983 \\
\text { Annual } \\
\text { Puel Cost } \\
\text { Sevingec } \\
\text { (\$) }\end{array}$} & \multicolumn{3}{|c|}{ Additionel 1983 Coste (\$) } & \multirow{2}{*}{$\begin{array}{l}\text { Approx. } \\
\text { Payback } \\
\text { Perlod (1f } \\
\text { evallable) }\end{array}$} & \multirow{2}{*}{$\begin{array}{l}\text { Aasumed } \\
\text { Market } \\
\text { Sectors }\end{array}$} & \multirow[b]{2}{*}{ Remarks } \\
\hline & & & & & Investment & $\begin{array}{l}\text { MaIn- } \\
\text { Cenance }\end{array}$ & other & & & \\
\hline \multicolumn{11}{|c|}{$\begin{array}{l}\text { EQUIPMENT PURCHASING } \\
\text { AND HODIPYING (Cont' }\end{array}$} \\
\hline & $\begin{array}{l}\text { Uae of dedicated piggy- } \\
\text { back (traller-on-11atcar } \\
\text { or contal ner-on-flatcar) } \\
\text { traine (Refs. 36-38) }\end{array}$ & $\begin{array}{l}\text { Up }_{\text {so }} \text { to } \\
\text { (eat.) }\end{array}$ & 20 & $\begin{array}{l}\text { TRS } \\
\text { (Revenue } \\
\text { gharing } \\
\text { with rat1- } \\
\text { road w111 } \\
\text { sloo occur) }\end{array}$ & $\begin{array}{c}\text { TRS } \\
\text { (see } \\
\text { Rewarks) }\end{array}$ & $\mathbf{0}$ & - & $N / A$ & $\begin{array}{l}\text { Intercity } \\
\text { trucks } \\
\text { coving } \\
\text { over } 200 \\
\text {-ile }\end{array}$ & 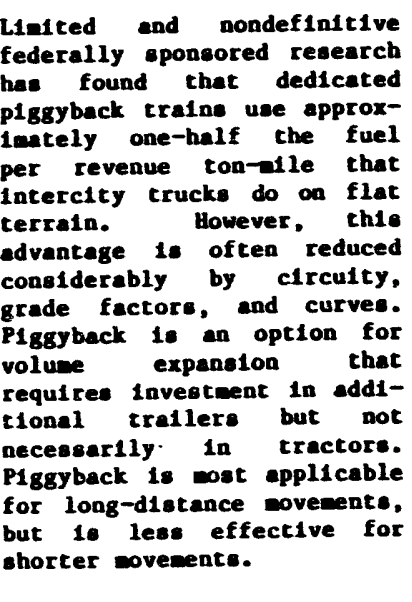 \\
\hline & $\begin{array}{l}\text { Une of vehiclea with } \\
\text { reduced tare weight } \\
\text { (Ref. 72) }\end{array}$ & $\begin{array}{l}\text { Up co } \\
5.5\end{array}$ & $\begin{array}{r}4 \\
20\end{array}$ & $\begin{array}{l}\text { Up to } 220 \\
\text { Up to } 1100\end{array}$ & $\begin{array}{l}\text { TRS } \\
\text { TRS }\end{array}$ & $\begin{array}{l}\text { TRS } \\
\text { TRS }\end{array}$ & $\begin{array}{l}\text { TRS } \\
\text { TRS }\end{array}$ & $\begin{array}{l}\text { TRS } \\
\text { TRS }\end{array}$ & $\begin{array}{l}\text { All trucks } \\
\text { All trucks }\end{array}$ & $\begin{array}{l}\text { We1ght reduction of up to to } \\
\text { 10x 1s posible, resulting } \\
\text { 1n 1ncreased payloade and up } \\
\text { to a } 5.5 x \text { fuel and ungs } \\
\text { according to one study. }\end{array}$ \\
\hline
\end{tabular}


Table 1 (Cont'd)

Per Truck

\section{Claimed}

\begin{tabular}{|c|c|c|c|c|c|c|c|c|}
\hline Claiped & & $\begin{array}{c}\text { Cla1med } \\
1983 \\
\text { Annual }\end{array}$ & Additional & 1983 Cost 8 & (\$) & $\begin{array}{l}\text { Approx. } \\
\text { Payback }\end{array}$ & Assumed & \\
\hline $\begin{array}{c}\text { Fuel } \\
\text { Savinge } \\
(z)^{\mathbf{z}}\end{array}$ & $\begin{array}{l}\text { Assumed } \\
\text { Puęl Useb } \\
\left(10^{3} \mathrm{gal}^{2} \mathrm{yr}\right)\end{array}$ & $\begin{array}{l}\text { Puel Cost } \\
\text { Savings } \\
\text { (\$) }\end{array}$ & Investment & $\begin{array}{c}\text { Main- } \\
\text { tenance }\end{array}$ & Other & $\begin{array}{l}\text { Period (1f } \\
\text { avallable) }\end{array}$ & $\begin{array}{l}\text { Market } \\
\text { Sectors }\end{array}$ & Rewarks \\
\hline
\end{tabular}

UEL AND LUBRICANT CHANGES

\section{Uae of gaseous fuel}

Uasoline

a. Propane (11quefied petroleus gas, or LPG

- total substitution

\begin{tabular}{|c|c|c|c|c|}
\hline $\begin{array}{l}\text { Subst1- } \\
\text { tutes } \\
\text { for } \\
\text { gasoline }\end{array}$ & 4 & $600-800^{h}$ & $\begin{array}{c}1000-1600 \\
\text { (conver- } \\
\text { sion) }\end{array}$ & $\begin{array}{c}0 \\
\text { (see } \\
\text { cautions } \\
\text { In } \\
\text { Rearks) }\end{array}$ \\
\hline
\end{tabular}

TRS
$(18$ months
claimed 1n
1n some
cases)

Local

plckup and

p1ckup and

and rout

dellvery

fleets

(> 15

gasollne-

powered

trucks in

fleet)

Not appllcable for dieseltrucks. Gas tank 1s replaced by pressure vessel. lequires buik storage cank at each fueling location or use of 8,000-10,000 propane tations (v8. 160,000 gasoline stations), resulting in lisited range. Mileage (apg) 18 approxiantely $5 x$ less, but lower propane costs regult la net savings of 15-20\%/gal (sose fleet: clain higher savings). Driver mat follow slightly different operacing procedures. Caution: Hot allowed in tunnels and certaln bridges and should always be parked outdoors due to possible leakage of volatile funes. Bxtreme care should be taken not to overfill tanks, because noraal expansion can activate the emergency pressure release valve and create fire hazard. Bleet demonstrations have shown lnaccuracles with propane fuel gauges. Maintenance of epark pluge, ignition, coollng, and aeration ystems aust be euperior to prevent detonation. How ever, less saintenance 1 required; for exanple, opark plugg and lubricating ol last 2 to 3 tines longer. 
Table 1 (Cont'd)

Per Truck

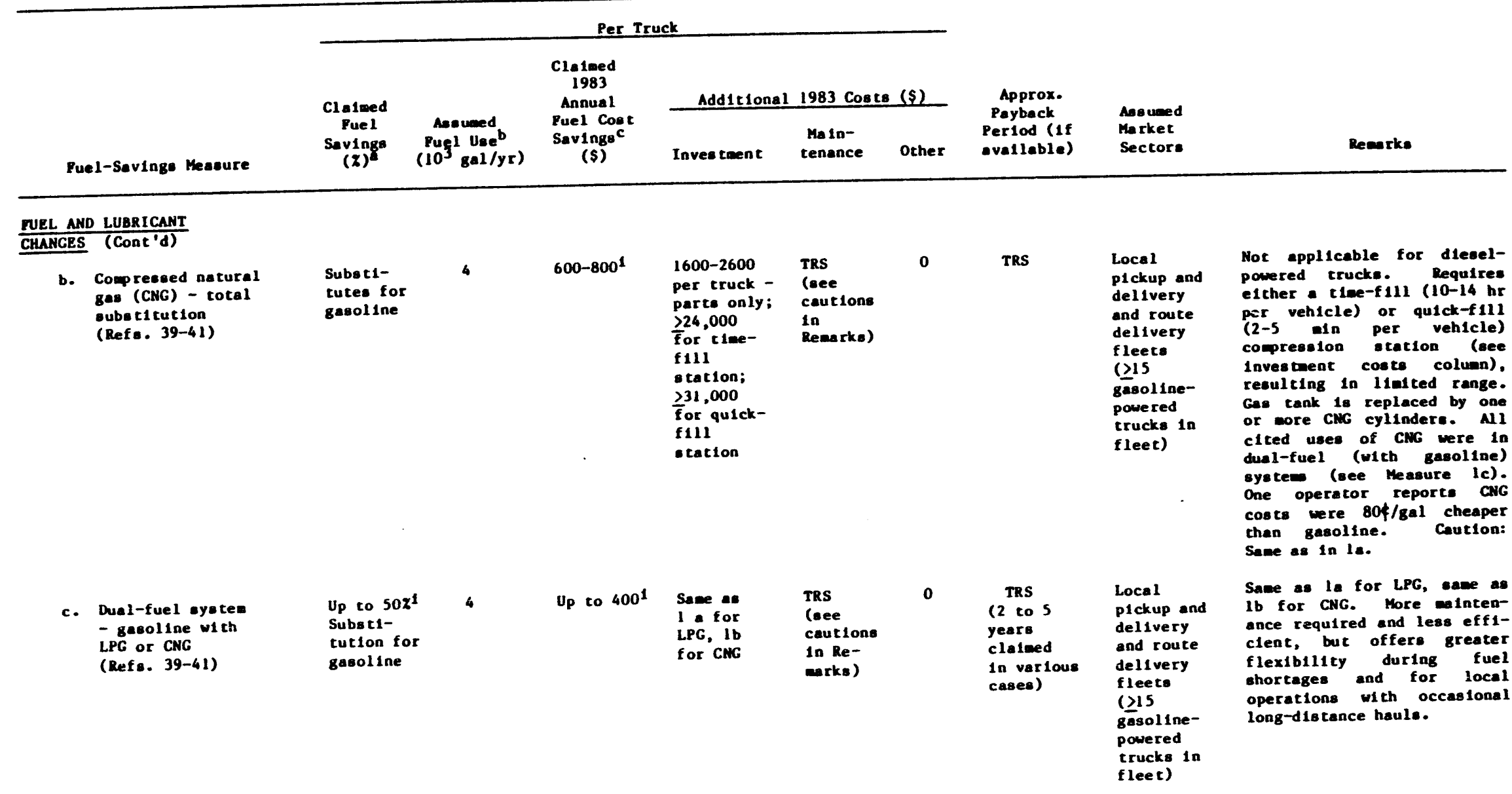


Table 1 (Cont'd)

Per Truck

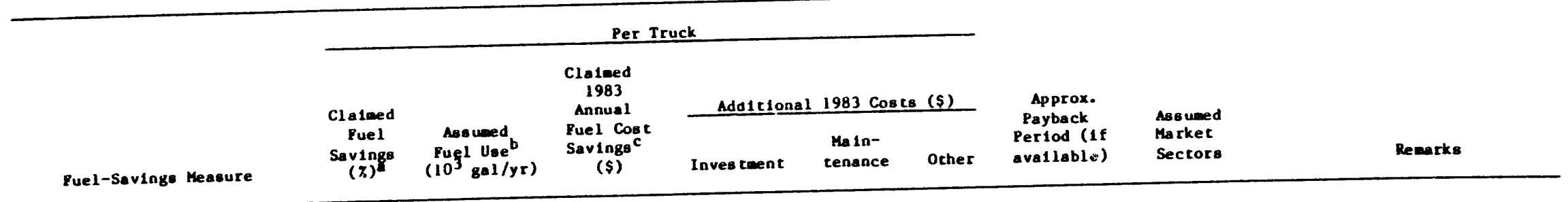

FUEL AND LUBRICANT

2. Use of extended-life engine lubricants

a. Synchetic (Ref B . 42-44)

Up to $3^{\mathbf{e}}$

4
20

Up to 120
Up to 600

(20)
petroleun-based

TRS

TRS

TRS

0

0
$\operatorname{TRS}^{\mathbf{e}}$

$\quad 0$
(h1gher
o1l cost
of f set
by fewer
o1l
changes)

Hore expensive (\$12/ga1) but al les (w1 th addition of some mke-up oll) va. 25,000 iles for conventional lubricants. Hoat benef iclal in colder climates; has 1mproved low-temperature fluidity for cold weather starting and engine protect1on. stability results in reduced oll degradation during service. Uidely used in U.S. and Canadian highway and of $f$-highway tleets. Generalizations should not be ade asong brands of synthetic lubricants.

Contains agents to reduce internal engine friction and additives to resist viscosity chenges and
inhibit oxidation and corrosion. 
Table 1 (Cont'd)

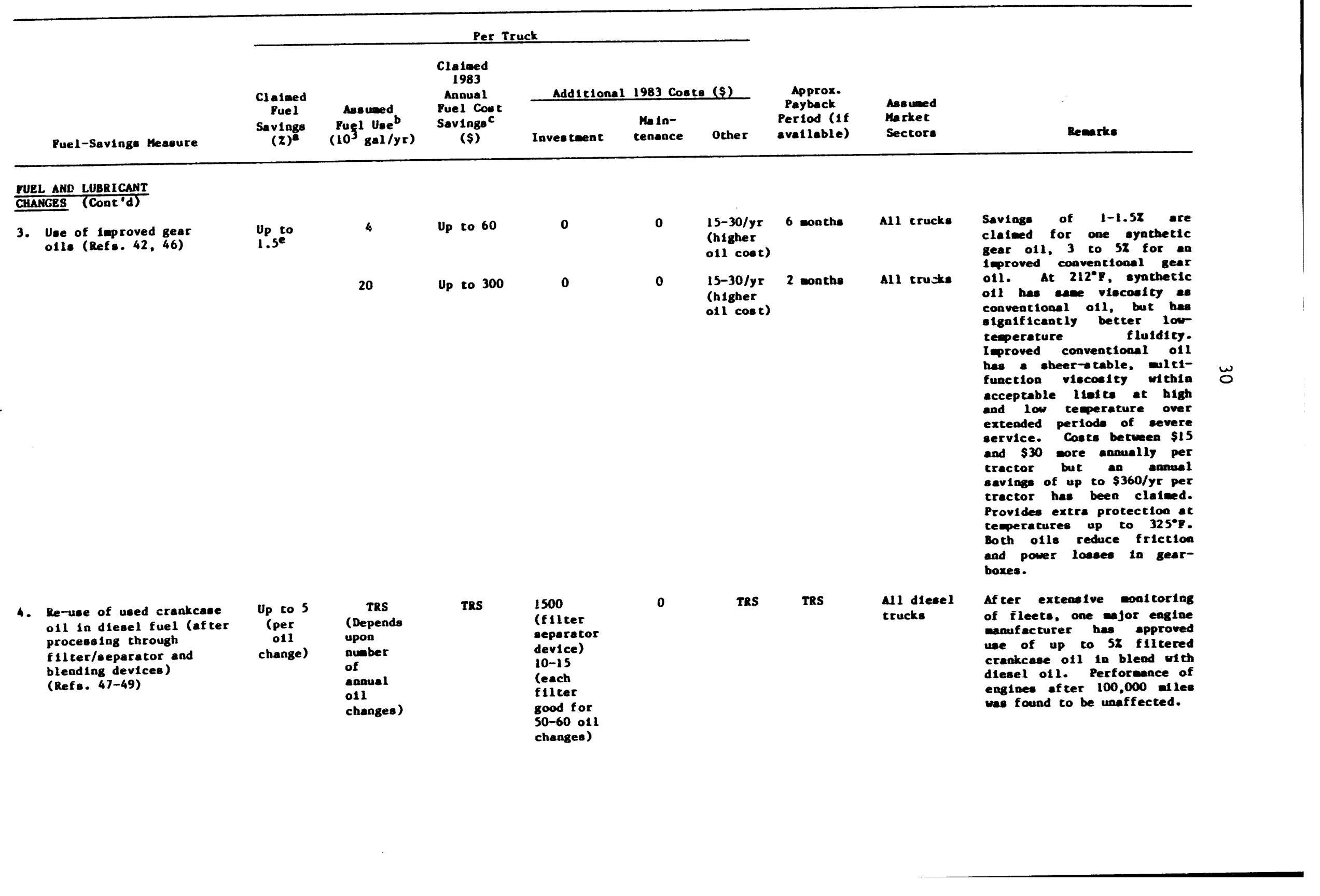


Table 1 (Cont'd)

Per Truck

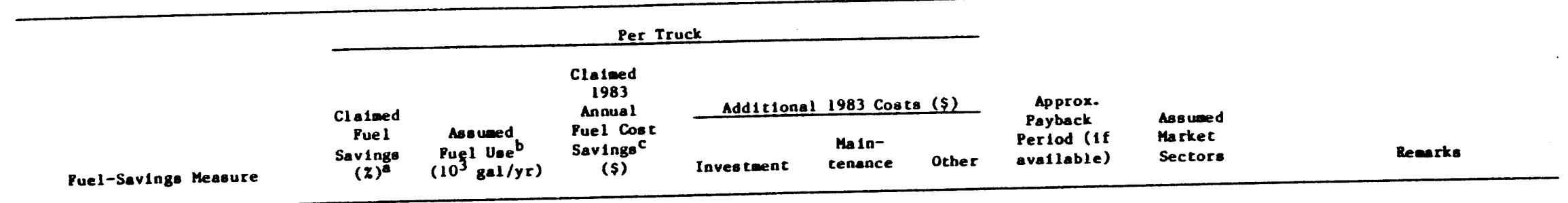

YURL AND LUBRICANT

CHANGES (Cont'd)

5. Use of alcohol fuels as extenders of petroleum based fuels

a. Hith gasoline - 108 blend (ganohol) (Refa. 41,50,51)

Puel
economy
17 (Sub-
otc cution
for gas-

TP.S

12 (Sub-

- ticution

for gas-
ollne 10x)

b. With diesel fuel

TRS

(d reselhol)
(Ref. SO)

TRS
TRS

o

o

0

N/A

All

gasollne-

powe red
trucks

0

0

N/A

Al1

diesel-

polvered
zrucks
Fuel efficlency is equal to or -l1ghtly better than atraight gasoline because of octane lncrease. Does not cause engive danage. One cause found that if ganohol 1s purchased for less than lof/gal core than the chespest gasoline a truck can use, cost savings will be obrained. Ethanol blends are core fuel efficient than those using thanol because ethanol has a higher emergs content.

Mo definitive findiags exist on net petroleu suevings: blesds are known to be lese fuel effictent than gasohol, because the lower eaergy conteat of alcohol is nor counter-balanced by 1 iproved conbustibility in diesel fuel-alcohol blend. Alcohol 1s not a cetane ipprover. 
Table 1 (Cont'd)

Per Truck

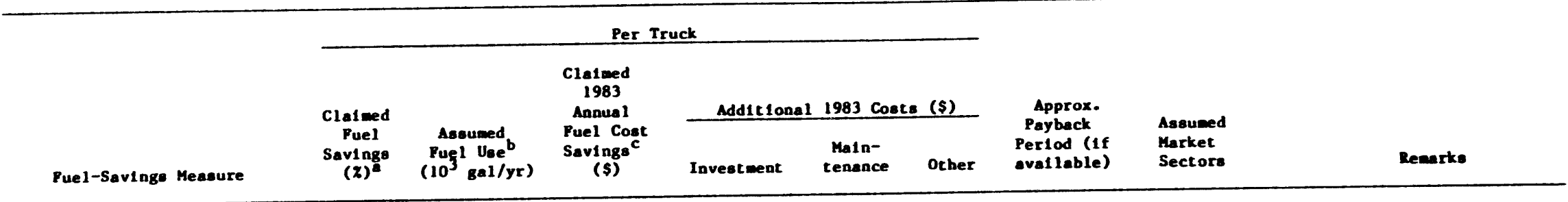

TECHNOLOGIES UNDER

DEVELOPARET

1. Organic Bank1ne bottoning-

cycle waste heat recovery

Up to 15

20

Up to 3000

$\$ 10,000$

Increase

In cost

eractor

$\begin{array}{lll}0 & \begin{array}{l}2 \text { years } \\ \text { (eat.) }\end{array} & \begin{array}{l}\text { All new } \\ \text { heavy-duty } \\ \text { Intercity } \\ \text { trucks }\end{array}\end{array}$

Road tests with 70,000-1bgrose tractor-traller have achleved 12.52 fuel anvings, but s1x wore years of testIng and demonstration are profected. Up to 40 addithoal horsepower are eat 1 ated to be achievable through recovery of waste beat (exhaust gas) in a 290horsepower engine. It 10 anticipated that a fev atandard designa will be adaptable to many different engines. Major component. Include turbine, gearbox, feed punp, vapor generator, condenser, and regenerator. could be comerclally avallable ta a feu years. 2. Gas turb1 ne engine
(Refs. 54-56,78)

Up to 100
(potent1al
oll savings
through
alternate

TRS

TRS

Unknown

Unknown

Unknown Unknown

All new

long-

distance

fuel use)

Twenty-four trucks have been ueed for operational teat in uidely divergent locat1ons throughout D.S. Puel consumption rate was once higher than diesel due to high fuel-flow rates at 1dle. Main energy saving potential lies in conbustion efficlency inprovenents (e.g., through development and use of high-temperature ceraencs) that enable use of alternate fuels (1.e., coni derived anfuel, ethanol fuels. Host applicable for 
Table 1 (Cont'd)

Per Truck

\begin{tabular}{|c|c|c|c|c|c|c|c|c|c|}
\hline \multirow{3}{*}{ Puel-Sevinge Measure } & \multicolumn{6}{|c|}{ Per Truck } & \multirow{3}{*}{$\begin{array}{l}\text { Approx. } \\
\text { Payback } \\
\text { Period (1f } \\
\text { avallable) }\end{array}$} & \multirow{3}{*}{$\begin{array}{l}\text { Assumed } \\
\text { Market } \\
\text { Sectors }\end{array}$} & \multirow[b]{3}{*}{ Remerks } \\
\hline & \multirow{2}{*}{$\begin{array}{c}\text { Claimed } \\
\text { Puel } \\
\text { Savings } \\
(x)^{g}\end{array}$} & \multirow{2}{*}{ 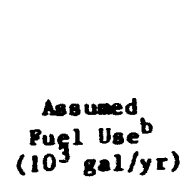 } & \multirow{2}{*}{$\begin{array}{l}\text { Clalaed } \\
\text { 1983 } \\
\text { Annual } \\
\text { Puel Cost } \\
\text { Savinge } \\
\text { (\$) }\end{array}$} & \multicolumn{3}{|c|}{ Addiclonal 1983 Costs $(\$)$} & & & \\
\hline & & & & Inves tment & tenance & Other & & & \\
\hline
\end{tabular}

TECHNOLOGIES UNDER

DEVELOPMENT (Cont'd)

3. Ocher energy recovery and

TRS

rRs

TRS

Uaknown

Unknown Unknown

fuel tolerance tech-

nologies (turbocomound

inime-friction adiabatic

engl ne; fully adiabatic

turbocomound diesel;

adiabatic engine plua

botronlag cycle; spark

1 gnited/assisted

tratif led-charge gaso-

line and diesel engines)

(Ref s. 54, 57, 58, 79) long-distance operations at ull pover mot of the cine. It 1s eetinted that auccesaful application in quantity will not occur unt11 1990-2005.

In various stages of research and development and projected for comerclallzac1on between 1985 and 2000. Turbocospound diesels have been under developent olace 1972 and use turbine pouver generated by exhaust gas expansion by craasferring the power to the drivetrain by gearing the curbine to the engine crankshaft. $50,000-1$ fleld test of curbocompound, turbocharged, and af tercooled engine on heavy-duty truck found fuel determined to be due to turbocomounding alone). Driver reaction was favor able to the quitet and smooth operation and strong per formance oa long grades. Turbocharger elficlency has been found co be critical of exhaust gas energy. Adlabatic engines are based on concept of Insulating diesel combustion chanber
with bigh-temperature 
Table 1 (Cont'd)

\begin{tabular}{|c|c|c|c|c|c|c|c|c|c|}
\hline \multirow[b]{3}{*}{ Puel-Savinge Measure } & \multicolumn{6}{|c|}{ Per Truck } & \multirow{3}{*}{$\begin{array}{l}\text { Approx. } \\
\text { Payback } \\
\text { Period (ff } \\
\text { evallable) }\end{array}$} & \multirow{3}{*}{$\begin{array}{l}\text { Aasumed } \\
\text { Market } \\
\text { Sectors }\end{array}$} & \multirow[b]{3}{*}{ Remrke } \\
\hline & \multirow{2}{*}{$\begin{array}{c}\text { Clalned } \\
\text { Puel } \\
\text { Savinge } \\
(\bar{x})^{8}\end{array}$} & \multirow{2}{*}{$\begin{array}{l}\text { Aesumed } \\
\text { Puefl Use } \\
\left(10^{3} \mathrm{gal} / \mathrm{yr}\right)\end{array}$} & \multirow{2}{*}{$\begin{array}{c}\text { Clained } \\
\text { 1983 } \\
\text { Annual } \\
\text { Fuel Cost } \\
\text { Savingec } \\
\text { (\$) }\end{array}$} & \multicolumn{3}{|c|}{ Additional 1983 Coste (\$) } & & & \\
\hline & & & & Inven tmer. $t$ & renance & Other & & & \\
\hline
\end{tabular}

TECHNOLOGLES UNDER

DEVELOFAENT (Cont'd)

4. Undercarriage wind

Unknown Unknowm

Unknown

Unknown Unknown

desiga (Ref. 69)

5. Injection of water and

lcohol blend 1n diesel
engines (Ref.. 79, 80,81)

Unknown Unknown Unknown Unknown Unknown Unknown Unknown All trucke 
Table 1 (Cont'd)

er Truck

\begin{tabular}{|c|c|c|c|c|c|c|c|c|c|}
\hline \multirow[b]{3}{*}{ Puel-Savingo Measure } & \multicolumn{6}{|c|}{ Per Truck } & \multirow{3}{*}{$\begin{array}{l}\text { Approx. } \\
\text { Payback } \\
\text { Perlod (1f } \\
\text { avallable) }\end{array}$} & \multirow{3}{*}{$\begin{array}{l}\text { Assumed } \\
\text { Market } \\
\text { Sectors }\end{array}$} & \multirow[b]{3}{*}{ Rewarks } \\
\hline & \multirow{2}{*}{$\begin{array}{l}\text { Claimed } \\
\text { Puel } \\
\text { Savings } \\
(x)^{8}\end{array}$} & \multirow{2}{*}{$\begin{array}{l}\text { Ass umed } \\
\text { Puģl Use } \\
\left(10^{b} \mathrm{gal}^{\mathrm{yr}}\right)\end{array}$} & \multirow{2}{*}{$\begin{array}{l}\text { Claimed } \\
1983 \\
\text { Annual } \\
\text { Puel Cost } \\
\text { Savingsc } \\
(\$)\end{array}$} & \multicolumn{3}{|c|}{ Add1tional 1983 Costs (\$) } & & & \\
\hline & & & & Inves twent & $\begin{array}{l}\text { Ma In- } \\
\text { tenance }\end{array}$ & other & & & \\
\hline$\frac{\text { TECHNOLOGIES UNDER }}{\text { DEVELOPMENT (CONT'd) }}$ & & & & & & & & & \\
\hline $\begin{array}{l}\text { 6. Electronic controls } \\
\text { a. Puel (Ref. 59) }\end{array}$ & Up to 7 & $\begin{array}{r}4 \\
20\end{array}$ & $\begin{array}{l}\text { Up to } 280 \\
\text { Up to } 1400\end{array}$ & Unknown & Unknown & Unknown & Unknown & $\begin{array}{l}\text { All diesel } \\
\text { trucks }\end{array}$ & $\begin{array}{l}\text { Controls belag bench-tested } \\
\text { Include a famf ly of pumps, } \\
\text { sensors, and microprocessors } \\
\text { to govern dellvery of fuel } \\
\text { to injectors and select } \\
\text { optiminection tining. }\end{array}$ \\
\hline b. Transm1ssion (Ref. 60) & Unknown & Unknown & Unknown & Unknown & Unknown & Unknown & Unknown & $\begin{array}{l}\text { All diese } 1 \\
\text { trucks }\end{array}$ & $\begin{array}{l}\text { Concrols are expected to } \\
\text { reduce shift-point varia- } \\
\text { tions fromoptimin from } \pm 75 \\
\text { rpo down to } \pm 15 \text { rpe. First } \\
\text { applications planned for } \\
\text { 1983-84. }\end{array}$ \\
\hline
\end{tabular}

Auel savings of the various asures are not directly additive because each new cavings will diminish the annual base of fuel consuaption that can be further reduced.

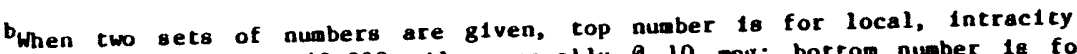
fleets and assumes 40,000 miles annually e 10 mg; bottom number la for entercity fleets and assumes $100,000 \mathrm{mllea}$ annually assume diesel operations.

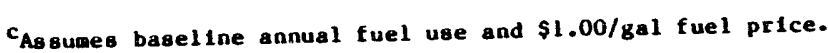

dTKS - Truck- and route-specific. Costs can Include managent progran and

e Reference estimates adjusted by Industry coordination meeting consensus.

$f_{N / A}$ - Not applicable.

Buses comparison of two engine types of a major manuf acturer.

hased on a gas company's estimated 15-20ł/gal net savings after 52 loss in mpg.

$1_{\text {Based on estinated use of LPG or CNG as substitute for gasoline dally (total }}$ substitution) or every other day (dual-fuel systea), and on a gas company's estimate of net potential savings of 15 to $20 / / g a l$ af ter 52 loss $1 \mathrm{n}$ apg.

ecterice could be found for a daca 1 tem. 
This section demonstrates the usefulness of the industry coordination meeting as a research tool for use in (1) developing and evaluating summary information and (2) identifying and discussing the specific revisions that Improved the matrix.

\subsection{SUMMARY OF INDUSTRY COORDINATION MEETING RESULTS}

One 1mportant result of the meeting was the unanimous agreement by the particlpants that the first measure in the matrix (1.e., development of driver understanding, motivation, cooperation, and participation, preferably through a management program) is the single most important part of any truck fuel savings program. The human element, namely the driver, was considered cructal to the success of fuel-saving equipment and procedures. It is not colncldental that the same generlc consensus regarding operating personnel was reached in the earlier Industry coordination meetings conducted by ANL during the last two years for the maritime and rallroad industries. At all these meetings, roughly the same percentage of participants agreed unanimously that this measure offers the greatest savings potential. These results testify to the pervasive importance of the human element in energy efficient transportation systems. the matrix:

Discussions during this meeting resulted in the following changes to

- There were 55 fuel-saving measures in the draft matrix; one was deleted and eight were added for a final total of 62 .

- The fuel-savings percentage estimates for five measures were revised.

- The payback period estimates for eight measures were changed.

- There were 17 substantive changes and/or additions to the remarks (e.g., advantages, disadvantages) columns of 14 measures. The meeting also resulted in the addition of 29 publications to the matrix reference 11st, increasing the total from 60 to 89 . 


\subsection{ADDITION AND DELETION OF MATRIX MEASURES}

The measures listed below were added to the matrix as a result of participant recommendations made during the coordination meeting. The participants also provided appropriate reference sources for use in developing both the summary data for the matrix and the reference list to provide a source of more detalled and extensive information. The added measures were:

- Maintain coolant temperatures.

- Adjust aerodynamic devices based on traller helght and gap.

- Install transmission with fewer fixed gear ratios and an overdrive for on-off highway, fixed-speed crulse operation.

- Use diesel turbocharged/aftercooled engine.

- Use regional break-bulk terminals.

- Use vehicles with reduced tare weight.

- Use undercarriage wind deflectors and improvements in aerodynamic design; injection of water-and-alcohol blend in diesel engines.

In the making of $s i x$ of these elght matrix changes, unlike those discussed in the subsequent sections, truck operators generally allowed the research community to take the lead. This is consistent with the approach generally taken by truck operators in considering changes to their operations and equipment, i.e., allowing the research and equipment-manufacturing communitles to offer fuel-savings alternatives. Although not as familiar with these measures as with the other 56, the Industry participants saw sufficlent merit in the information provided orally by the research community members to accept them as additions to the matrix pending AN review of the cited references. This review verified the oral information presented at the meeting. Significantly, however, no specific fuel-savings percentage estimates were agreed on for any of the added measures, although the results of some studies and tests were included in the "Remarks" column of the matrix.

Two of the first six added measures were proposed by Raymond Camball, project manager of the Canadian TRUCKSAVE Program, which operates in Ontario. This resulted not only in these two new measures (which were not found in U.S. truck research literature by $A N L$ ), but also in nine new references that are included in Appendix A.

John Burke, a former official of the U.S. Voluntary Truck and Bus Fuel Economy Program, proposed the transmission options and diesel 
turbocharged/aftercooled engine measure, as well as the undercarriage wind deflectors and improved aerodynamic tractor designs. He also supplied 18 new references that were added to the list in Appendix A.

Professor McNally proposed adding to the list of technologies under development the research and testing being done on infecting a water-andalcohol blend into diesel engines. This proposal was also accepted, with particular reference made in the matrix to similar research and development in the maritime industry.

Finally, two new measures in the above list were suggested by truck industry representatives. One measure was added when truck operators pointed out that regional break-bulk terminals en route for intercity movements are analogous to city consolidation terminals and are already being used to increase cargo ton-miles per mile driven. The second measure was added when the operators cited examples of vehicles with reduced tare weight that are already avallable and being used to increase load factors.

\subsection{REVISIONS OF FUEL-SAVINGS PERCENTAGE ESTIMATES}

Al1 recommendations for revisions in fuel-savings percentage estimates at the meeting were inttiated by truck operators, based on their operating experiences. These recommendations, combined with the operators' agreement with the rest of estimated percentages in the matrix, were among the most important contributions made by industry. The revisions were made to include a perspective based on a wider cross-section of real-world truck operations to the fuel-savings estimates that were based on research and/or limited tests. The five revisions of fuel-savings percentage estimates are as follows:

- Improve driving practices: 1 to $10 \%$ changed to Up to $12 \%$ for both intercity and intracity operations.

- rose-couple tractor and trailer and/or use side panels between traller and container combinations: Truck- and route-specific changed to $U p$ to $5 \%$ for intercity operations.

- Install improved alr Intake and exhaust system equipment: Up to $3.7 \%$ changed to Up to $2.5 \%$ for both intercity and intracity operations.

- Use of synthetic extended-1ife engine lubricants: Item 2a; Up to $6 \%$ changed to Up to $3 \%$ for both intercity and intracity operations.

- Use of improved gear ofls: 1 to $5 \%$ changed to Up to $1.5 \%$ for both intercity and intracity operations. 
The downward adjustment of three estimates and upward adjustment of two reflected the levels of success encountered by Industry participants in employing the various measures.

\subsection{ADJUSTMENTS OF PAYBACK PERIOD ESTIMATES}

The payback period, or length of time required for an investment to save a company enough money to pay for itself, is an important criterion to a business contemplating that investment. The adjustments made to paybackperfod column for elght measures at the suggestion of industry particlpants were valuable contributions. Six of the elght adjustments were favorable, Indicating that industry participants have had very positive experiences with several measures. The elght adjustments were as follows:

- Development of driver understanding, motivation, cooperation, and particlpation (preferably through a management program): Truck- and route-specific changed to Less than six months for both intercity and intracity operations.

- Supervise outside fuelings to avold "overfills": Changed from Not Applicable to Immediate for both intercity and intractity operations.

- Install aerodynamic devices (e.g., wind deflector tractor cab, bubble on traller): Less than one year changed to six months for interclity operations.

- Close-couple tractor and traller and/or use side panels with trailer and contalner comblnations: Truck- and routeopecific changed to include May be immediate in parentheses for intercity operations.

- Install tachometers: No estimate changed to Less than one year for both interclty and intraclty operations.

- Install tachographs: No estimate changed to Less than one year for both intercity and intracity operations.

- Use of synthetic extended-1ife engine lubricants: No payback period changed to Truck- and route-specific for both intercity and intracity operations.

- Use of extended-11fe engine lubricants that are multiviscosity, petroleum-based: Less than six months changed to Truck- and route-specific for both intercity and intracity operations of diesel trucks. 


\subsection{SUBSTANTIVE CHANGES TO REMARKS COLUMN}

Because of the many (17) substant1ve changes to the "Remarks" column (e.g., advantages, disadvantages, technical explanations of measures) for 14 matrix measures, this section concentrates only on elght selected changes in the interest of brevity. The changes discussed are individual comments or sets of comments ragarding a fuel-savings measure. They are anong the most Important changes made to this colum in terms of new and/or clarifying information provided.

The first set of changes in the "Remarks" column was for the measure calling for development of driver understanding and support of a fuel savings program. Whether or not a formal management program is used, meeting particlpants agreed that the setting of realistic goals (e.g., achievable Improvements in mpg) is 1mportant to successful fuel-savings efforts. Realistic goals aid these efforts by not only providing sufficient opportunities for positive reinforcement through the reaching of goals, but also help prevent the cheating that has been known to occur, according to meeting participants. The particlpants also recommended adding two examples of successful program elements for fleet operations based upon their experiences, 1.e., (1) use of an advisory board composed of drivers to select new drivers and (2) use of a team incentive program to add peer pressure to financial incentives in improving fuel efficiency. Such driver group activities were considered to be useful in bullding teamwork and sharing information among drivers.

The second important change to the "Remarks" column was made for the 55-mph maximum speed measure. A consensus statement was added to note the particular importance of not exceeding $55 \mathrm{mph}$ when headwinds are high, because disproportionately high power increases are required.

A statement was added for the measure calling for avoldance of sudden stops and starts. It was noted that this measure requires careful observation of other vehicles and choosing of a lane that will let the truck keep moving while maintalning an adequate distance from the vehicle ahead. This element of driving strategy is necessary, particulariy on heavy traffic routes, if the measure is to be accomplished.

The fourth added remark was for the measure that calls for monitoring of fuel consumption. Meeting participants pointed out that electronic monitoring instruments are good driver-training devices even if there is not enough capital to equip the entire fleet.

The fifth comment added an operational (1.e., service) incentive for the use of satellite terminals in large cities. The reminder placed in the remarks column (noting that the reduction in stem times effected by the terminals is often more valuable than the fuel savings achieved) provides trucking firms with an even greater reason to use satellite terminals. 
The sixth set of changes was related to radial tires and their safe and proper use. A caution was added for the recently developed "super-single" radials (1.e., single tires that replace dual radials, reducing weight and rolling resistance). It was noted that they do not handle well on wet roads at high speeds. In addition, recommendations were made for better alignment programs for radial tires than for bias ply tires on all free-rolling axles and for maximization of coasting. The latter was recommended because radials coast up to $30 \%$ more effectively than blas-ply tires and this fact should be demonstrated to drivers during training. These inclusions in the "Remarks" column provide to the novice user of highly popular radials valuable information not found in the many references that describe radials and that were used to develop the draft matrix.

The seventh added remark concerned installation of fan clutches. While some early users may have found these devices to be unreliable, the recent experiences of the meeting participants resulted in the addition of a remark indicating that fan-clutch reliability has improved significantly in recent years.

The elghth set of changes significantly altered the remarks concerning the use of propane (1iquefled petroleum gas, or LGP) rather than gasoline. The meeting consensus was that the draft matrix remarks taken from the literature unduly emphasized the dangers of using propane. Noting, for example, the extreme combustibility of gasoline and its harmfulness to the skin, participants removed statements cautioning that the "engine must not be started and then shut down without giving the coolant a chance to attain $55^{\circ} \mathrm{F}$ " and that "propane contact with the skin causes serlous freeze burns." These were replaced with a statement that the "Driver must follow slightly different operating procedures." Regarding maintenance, the participants retained a draft matrix comment on the need for careful attention to spark plugs, ignition, cooling, and aeration systems to prevent detonation. However, to give proper credit to the maintenance advantages of propane, the participants agreed to add a statement that "...less malntenance is required; for example, spark plugs and lubricating ofl last two to three times longer." Finally, participants' respects for the dangers of using propane is demonstrated by the comment added to the remarks that "extreme care should be taken not to overfill tanks, because normal expansion can activate the emergency pressure release valve and create a fire hazard." These changes, although not related to fuel efficiency, provide operators who contemplate the use of propane with balanced information on its advantages and disadvantages. The changes downplaying the dangers of propane were included in the matrix because they came from truck operators, not from propane manufacturers. 


\section{CONCLUSIONS}

This report contalns a compendium of information developed for the trucking Industry on options avallable for saving fuel. The considerable research value of an industry coordination meeting in revising the matrix was focused upon and clearly demonstrated. Moreover, the credibility of this Information dissemination program with its intended audience was greatly enhanced by describing participants' input and providing a list of the particlpants as part of a malling list sent to members of the trucking 1ndustry. An 1mportant result of this program was the consensus reached at the meeting, as at the maritime and railroad industry meetings, that the vehicle operator is the single most important ingredient in a successful fuelsaving program. 
APPENDIXES 
APPENDIX A: MATRIX REFERENCE LIST

1. U.S. Jolnt Industry-Government Voluntary Truck and Bus Fuel Economy Program, How to Save Truck Euel, U.S. Departments of Transportation and Energy and U.S. Environmental Protection Agency, DOT-HS-803-768, (November 1980; revised edition October 1982). Coples of 1982 edition are avallable from Motor Vehicle Manufacturers Association, Washington, D.C.

2. Cross, R., MPG: The Human Factor, Commercial Car Journal, pp. 80-85 (November 1979).

3. Dwyer, J.J., Executive Decision at the Throttle, Fleet Owner, pp. 51-57 (December 1977).

4. Winsor, J., and R. Warner, Driver Professionalism = Productivity Profits, Performance, Commerclal Car Journal, pp. 99-103 (March 1977).

5. Bald, J., Drivers Earn What They Don't Burn, Fleet Owner, pp. 76-78 (January 1980).

6. Who or What Controls an Engine's Temperature, Fleet Owner, pp. 86-87 (Apri1 1980).

7. Selff, H.E., The Economics of Better Economy for Owner Operations, paper presented at Soclety of Automotive Engineers West Coast Meeting, San Francisco (August 9-12, 1976).

8. Cope, E.M., The Effect of Speed on Truck Fuel Consumption Rates, report prepared for Federal Highway Administration, U.S. Department of Transportation (August 1974).

9. Duncan, T.W., Can We Force 55? Fleet Owner, Pp. 79-83 (April 1978).

10. U.S. Joint Industry-Government Voluntary Truck and Bus Fuel Economy Program, The Double Nickel Challenge, U.S. Department of Transportation, DOT-HS-804-097 (May 1979).

11. Preston Trucking Company, Driver Instruction Sheet, Preston, Md. (undated).

12. Johnson, L.R., et al., Fuel Conservation and Contingency Planning, in Goods Transportation In Urban Areas (Proc. Engineering Foundation Conf., Easton, Md., June 1981), G.P. Fisher and A.H. Meyburg, eds., U.S. Dept. of Transportation Report UMTA-NY-06-0087-82-2, pp. 51-68 (Jan. 1982). 
13. Schuster, A.D., Fusl Conservation in Urban Goods Distribution, In Goods Transportation in Urban Areas (Proc. Engineering Foundation Conf., Easton, Md., June 1981), G.P. Fisher and A.H. Meyburg, eds., U.S. Dept. of Transportation Report UMTA-NY-06-0087-82-2, pp. 351-369 (Jan. 1982).

14. What You Should Know About E.I.A., brochure printed by Equipment Interchange Association, Washington, D.C. (undated).

15. Anderson, D.L., Deregulation Impacts on Truok and Trailer Sales in 19808, paper presented at Transportation Research Board 1981 Annual Meeting, Wash1ngton, D.C. (January 14, 1981).

16. Henfum, S.E., Radials Pass the PUD\&D Test, Fleet Owner, pp. 71, 72 (November 1980).

17. Lyndal1, J., Rolling Resistance vs MPG, Fleet Owner, p. 78 (November $1980)$.

18. Gross, R., Radials: Who Uses What - Where and Why, Commerclal Car Journal, pp. 94-101 (July 1980).

19. U.S. Jolnt Industry-Government Voluntary Truck and Bus Program, Study of Potential for Motor Vehicle Euel Economy Improvement - Panel Report No. 7, U.S. Department of Transportation and U.S. Environmental Protection Agency (January 1975).

20. Slegel, S., Pepsi Joins the Diesel Generation, Fleet Owner, pp. 75-77 (September 1979).

21. Galligan, J., What's Cooking with Diesels and Small Trucks, Commercial Car Journal, pp. 107-110 (March 1980).

22. Ritchie, D., Small Diesels for Small Trucks - Do They Have a Future? Commercial Car Journal, pp. 121-125 (March 1977).

23. Jacobs, K.M., Energy Usage and Other Comparisons Between Diesel and Gasoline Pickup Trucks, paper presented at Transportation Research Board 1979 Annual Meeting, Washington, D.C. (January 17, 1979).

24. Drive, Tag Tandem Axles Close in Euel Economy Tests, Automotive News, pp. 16-17 (November 23, 1981).

25. Mack Tries New Diesel, Turbine Engines, The Washington Post, p. 12 (March 12, 1981).

26. Formula 300 Engine Does 5.9 MPG in Field Test8, Fleet Owner, Pp. 52, 53 (September 1979). 
27. A Common Sense Approach to Fuel Boonomy, The Spec1al1st, pp. 55-56 (May/June 1980).

28. Amertcan Trucking Assoclattons, Inc., American Tmucking and the Energy Crisis, Washlngton, D.C. (1973).

29. An Investigation of Truck Sise and Weight Limits: Appendices, U.S. Department of Transportation, p. F-66 (August 1981).

30. Kollns, R.W., Truck Sise and Weight Limits: Their Impaots on the General Freight Common Carrier Costs and Market, Proceedings of Transportation Research Forum 18th Annual Meeting, Atlanta, Ga., pp. 323-332 (October 17-19, 1977).

31. Marlin, J.C., S.K. Dragula, and D.W. Marlin, Increased Truck Size and Weight; The Impacts on Highways, Safety and Energy, Central States Research Forum, Urbana, I11. (March 1980).

32. Encisco, B., Fuel and Labor Savings Through Greater Truok Sizes and Weights, Consolidated Frelghtways, Inc., Menlo Park, Callf. (February 1978).

33. Duncan, T.W., Triples: The Only way to Go, Fleet Owner, pp. 64-68 (Apr11 1980).

34. Hutton, T.D., Why Not Triples?, presented at Soclety of Automotive Engineers, West Coast Meeting, Anahe1m, Calif. (August 12-16, 1974).

35. DeYoe, E., Inoreasing the Payload - The Point of Mors Return, Fleet Owner, pp. 63-67 (Apr11 1977).

36. Bertram, K.M., Projected Potential Piggyback Energy Savings through the Year 2000, Transportation Research Record 801: Energy Data and Transportation, Transportation Research Board, National Academy of Sclences, Washington, D.C. (1981).

37. Intermodal Fuel Consumption Comparisons, Memorandum, Office of Transportation Programs, U.S. Department of Energy (January 9, 1980).

38. General Motors Transportation Systems Center, unpublished data developed wth computer simulations in U.S. Federal Rallroad Administration Intermodal Frelght Program, Warren, M1ch. (March 1980).

39. The Push to Propane: Can It Fit Into Your Fleet? Commerclal Car Journal, pp. 65-69 (December 1980).

40. Cross, R., The New Popularity of Gaseous Fuels, Commercial Car Journal, pp. 55-60 (December 1980). 
41. Southwest Research Inst1tute, Emergenoy Eusls Utilisation Guidebook: Altemative Fuels Utilisation Program, Division of Automotive Technology Development, U.S. Department of Energy (August 1980).

42. Keller, J.A., N.S. Kotuszenko, and R.R. McCoy, Fuel Economy Benefits of Synthetio Lubricants in Heavy Duty Truaks, Mob11 011 Corporation, New York (November 1979).

43. Saving Fuel is Really For the Birds, Commerclal Car Journal, pp. 138-141 (May 1979).

44. Two Shows, One Concern: Eusl Economy, Fleet Owner pp. 88-90 (Apr11 1978).

45. Extended Drain Oils Stretch Fleet Dollars, Fleet Owner pp. 60-62 (December 1980).

46. Lynda11, J., Gear Oil Can Help Save Fubl, Fleet Owner, p. 71 (September 1979).

47. Lyndal1, J., Engine Oil - The Second Time Around, Fleet Owner, pp. 84-86 (August 1979).

48. Racor Blender Mixes Used Oil in Diesel Oil, Fleet Owner, p. 52 (August 1979).

49. Lubs Oil Double Take, Fleet Owner, pp. 75-77 (December 1977).

50. Harnessing Alternative Euels, Fleet Owner, pp. 74-79 (August 1979).

51. Ryder Program in Transportation, University of Miami and Escher Technology Associates, St. Johns Mich., Altermative Fuels for Intercity Truoking, report prepared for Transportation Energy Conservation Division, U.S. Department of Energy (June 1978).

52. Thermo Electron Corporation, Preliminary Comercialization Plan - Rankine Bottoming Cyole Engine for Diesel Truoks, Waltham, Mass. (January 1981).

53. Koplan, M.D., and L. DiNanno, Thermo Electron Corporation, Status Report on Diesel Organic Rankine Compound Engine for Long Haul Trucks, ProceedIngs of Automotive Technology Development Contractors Coordination Meeting, Office of Transportation Programs, U.S. Department of Energy, pp. 252-257 (September 1981).

54. Whiteside, D., Technology: Key To The New Engines, Fleet Owner, pp. 5659 (January 1980). 
55. Jacobson, D.H., Heavy-Duty Engines Analysis, Study 3: Applications of Gas Turbine Engines for Heavy-Duty Trucks, Argonne Nat1onal Laboratory Report ANL/CNSV-TM-75, Argonne, I11. (July 1981).

56. Rockwood, F.A., Ceramic Applications in Turbine Engines, Proceedings of Automotive Technology Development Contractors Meeting, Office of Transportation Programs, U.S. Department of Energy, pp. 91-106 (September 1981).

57. Werner, J.R., Report on Vehicle Testing of the Cummins Turbocompound Diesel Engine, Proceedings of Automot1ve Technology Development Contractors Meeting, Office of Transportation Programs, U.S. Department of Energy, pp. 258-262 (September 1981).

58. Bryzik, W., Adiabatic Engine Program, Proceedings of Automotive Technology Development Contractors Meeting, Office of Transportation Programs, U.S. Department of Energy, pp. 269-281 (September 1981).

59. Electronics: More Precise Fuel Control, Fleet Owner pp. 71-74 (June 1981).

60. Electronics: Transmission Control, Fleet Owner, pp. 75-76 (June 1981).

61. Trucksave Program, Easy Going - Pro Driving for the $80 \mathrm{~s}$ and Beyond, pamphlet published by Ontarto Ministry of Transportation and Communication and Ministry of Energy, Downsview, Ontar1o, Canada (undated).

62. Calder, S., Truck Fuel Savings Programs, presented for the Trucksave Program at the Canadian Fleet Malntenance Seminar, Toronto (May 1982).

63. Trucksave Program, Idling Costs, pamphlet published by Ontario Ministry of Transportation and Communication and Ministry of Energy, Downsview, Ontarlo, Canada (undated).

64. Trucksave Program, Saving Truck Fuel: 2 Case Histories, pamphlet published by Ontario Ministry of Transportation and Communication and Ministry of Energy, Downsulew, Ontarlo, Canada (undated).

65. Cummins Engine Company, Inc., Guide to LCPM, Columbus, Ind. (Sept. 1981).

66. Trucksave Program, Cronkwright Takes Up Challenge, Trucksave News, Vol. 1, Issue 2, pp. 1,3, published by Ontarlo Ministry of Transportation and Communications and Ministry of Energy, Downsview, Ontario, Canada (Feb. 1982).

67. Trucksave Program, Engine Cooling Systems for Fuel Economy, pamphlet published by Ontario Ministry of Transportation and Communication and Ministry of Energy, Downsview, Ontario, Canada (undated). 
68. Trucksave Program, Speo'ing a Fuel-Efficient Truck, set of booklets published by Ontario Ministry of Transportation and Communication and Ministry of Energy, Downsulew, Ontario, Canada (April 1982).

69. Cumnins Englne Company, Inc., Automotive Technical Sales Seminar: Truck Performance and Euel Economy, Columbus, Ind. (July 1982).

70. "Super Singles": Tire of the Future?, Heavy Duty Trucking, pp. 61-63 (Aug. 1982).

71. U.S. Joint Industry-Government Voluntary Truck and Bus Fuel Economy Program, Computer Says Road-Speed Governor Saves Fuel, U.S. Department of Energy, pp. 6-7 (June 1979).

72. Energy Systems Planning Division, TRW, Inc., Potential Energy Savings in the Light-Vehicle, Long-Haul-Truck, and Bus Market Sectors, report prepared for Office of Planning and Pollcy, Office of Assistant Administrator for Conservation, U.S. Department of Energy (Nov. 1977).

73. Air Pressure and Fuei Consumption, Fleet Maintenance and Specifying, pp. 45-47 (Apr11 1981).

74. U.S. Joint Industry-Government Voluntary Truck and Bus Fuel Economy Program, Twin $45^{\prime} \mathrm{s}$ and Higher Weight Save $41 \%$ on Fuel in South Dakota Test, U.S. Department of Energy, p. 3 (Dec. 1980).

75. Ontar1o Government Drfve Propane Program, Switching to Propane: A Guide for Fleet Operators, guidebook published by Ontario Ministry of Transportation and Communication and Ministry of Energy, Downsulew, Ontario, Canada (Aug. 1981).

76. Ontario Government Drive Propane Program, Preliminary Summary of Fleet Demonstration Results, Ontarlo Minlstry of Transportation and Communication and Ministry of Energy, Downsview, Ontario, Canada (March 1982).

77. Voluntary Truck and Bus Fuel Economy Program, The Fuel Forrum: Diesel Organic Rankine Compound Engine (Bottoming yycle), Fuel Economy News, U.S. Departments of Transportation and Energy, pp. 5-6 (Dec. 1978).

78. Voluntary Truck and Bus Fuel Economy Program, U.S. Department of Energy Gas Turbine Bus Program, Fuel Economy News, U.S. Departments of Transportation and Energy, pp. 5-6 (March 1979).

79. Covey, R.R., and W.V. Roessler, Assessment of the Texaco ControlledCombustion System for Use in Light and Duty Vehicles, report prepared by Aerospace Corp. for Technology Development and Analysis Division, office of Vehicle and Engine Research and Development, U.S. Department of Energy (November 1982). 
80. M\&W Gear, Inc., M\&W Water/Alcohol Injection in Diesel Engines, c1rcular printed by M\&W Gear, Inc., Gibson C1ty, I11. (August 1979).

81. Mitsuhas1, K., et al., Application of Emulsified Fuel on Diesel Engine, Japan Shipbuilding and Marine Engineering, 13(1) (1979).

82. J.J. Keller and Associates, Reducing Costs Through Fuel Economy, Motor Carrier Safety Rep srt, VI(11), Neenah, Wis. (November 1979).

83. J.J. Keller and Assoclates, Plan Your Fuel Economy Program, Motor Carrier Safety Report, VI(7), Neenah, Wis. (July 1981).

84. U.S. Joint Industry-Government Voluntary Truck and Bus Fuel Economy Program, Fuel Economy News, U.S. Departments of Energy and Transportation (June 1981).

85. Gross, R., and W. Tracy, Fuel Conservation from the Drivers Seat, Commercial Car Journal, pp. 81-86 (November 1980).

86. U.S. Jolnt Irdustry-Government Voluntary Truck and Bus Fuel Economy Program, Truckers Guide to Fuel Savings, U.S. Department of Transportation, DOT-HS-805256 (Apr11 1981).

87. Saltzman, E.J., A Sumary of NASA - Dryden's Tmuck Aerodynamic Research, presented at 1982 Society of Automotive Engineers Truck and Bus Meeting Exposition Program (SAE Paper 821824), Indianapolis (November 1982).

88. Cooper, K.R., Wind Tunnel Testing of Heavy Trucks to Reduce Euel Consumption, presented at 1982 Soclety of Automotive Engineers Truck and Bus Meeting Exposition Program (SAE Paper 821825), Indianapolis (November 1982).

89. Buckley, F.T., Jr., Aspects of Over the Road Testing of Truck Aerodynamic Drag Reducing Devices, presented at 1982 Society of Automotive Engineers Truck and Bus Meeting Exposition Program (SAE paper 821286), Indianapolis (November 1982). 


\section{APPENDIX B: MEETING PARTICIPANTS}

\author{
Ken Penaluna \\ Vice President - Purchasing \\ Ruan Trucking \\ Des Molnes, Iowa \\ Robert L. Lyons \\ Driver Trainer \\ Preston Trucking Company, Inc. \\ Preston, Md. \\ Regina Selva \\ Department of Economics and Planning \\ American Trucking Assoctations, Inc. \\ Washington, D.C. \\ Thomas Dvorak \\ Distribution Services \\ Beatrice Foods Co. \\ Chicago \\ Richard Eads \\ Corporate Manager - Logistics \\ CFS Continental \\ Chicago \\ John Mande1 \\ Fleet Maintenance \& Specifying \\ Irving-Cloud Publishing Company \\ Lincolnwood, Ill. \\ Clifford Schoettmer \\ Southern Fleet Manager \\ Growmark, Inc. \\ Bloomington, Ill. \\ William Meehan \\ Manager, Transportation Operations \\ W.W. Grainger, Inc. \\ Ch1cago
}

John Burke

Former Sentor General Engineer

U.S. Voluntary Truck and Bus Fuel

Economy Program

Rockville, Md.

Daniel N. McNally

Professor, Dept. of Mechanical

Engineering Technology

Purdue University

West Lafayette, Ind.

Raymond Camball

Project Manager, TRUCKSAVE Program

Ontario Ministry of Transportation

and Communication

Downsview, Ontar1o

Thomas Mannix

President

Transportation Advisors, Inc.

Hollywood, Fla.

Kenne th M. Bertram

Project Manager

Center for Transportation Research

Argonne National Laboratory

Argonne, Ill.

Chris topher L. Saricks

Project Manager

Center for Transportation Research Argonne National Laboratory

Argonne, Ill. 
APPENDIX C: ARGONNE PRESS RELEASE

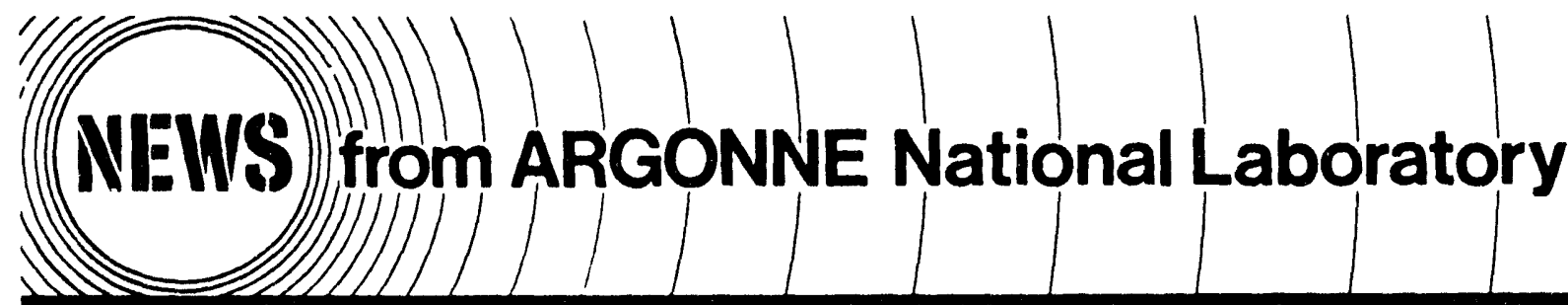

Office of Public Affairs • Argonne, Illinois 60439 - Telephone 312, 972.5575

December 15,1983

83-92

FOR IMMEDIATE RELEASE

Media contact: David Baurac

ARGONNE, ILL. ... The individual truck driver is the key to saving energy in the trucking industry. This is the conclusion of a recent study by the Department of Energy's Argonne National Laboratory and reflects the consensus reached at a trucking-industry meeting sponsored by Argonne during the study.

The study gathered and summarized information on more than 60 different energy-saving measures suitable for all truck operators. Measures included changes in operating procedures, add-on equipment. purchase of new vehicles with new fuel-saving features, fuel and lubricant changes, and new technologies under development.

"The role of the driver is crucial to the success of fuel-saving programs," said Ken Bertram of Argonne. Bertram is head of the study group. "New equipment and procedures to save fuel are most effective when individual drivers understand them and how they work and are strongly motivated to use them properly."

Specific fuel-saving measures included:

o Suostituting propane for gasoline fuel.

- Installing aerodynamic wind deflectors on top of tractor cabs.

o Buying new vehicles with high-torque, low-rpm diesel engines. 
- Using organic Rankine engines that reclaim exhaust heat and convert it into more hor sepower.

- Better and more timely maintenance.

Representatives of six firms with ongoing truck fuel efficiency programs and eight members of the private, academic and government truck research community attended an Argonne-sponsored, industry-coordination meeting in late 1982. The participants reached the same conclusions, while helping to refine Argonne's summary information. Organizations represented at the meeting were the American Trucking Associations, Beatrice Foods Co., CFS Continental Inc., H. W. Grainger Inc., Growmark Inc., Preston Trucking Co. Inc. and Ruan Trucking Inc.

The study's conclusion echoes the results of earlier Argonne studies of the maritime and rallroad industries. These studies held similar industry-coordination meetings that concluded that the key to successful energy-saving programs in those industries was the understanding and motivation of individual ship and railroad operating crews.

Argonine has prepared a chart summarizing the information available on more than 60 energy-saving measures that can be applied to local and long-haul trucking. Similar charts are avallable for the maritime and railroad industries.

Anyone interested in coples of the charts may contact Ken Bertram at the Center for Transportation Research, Building 362, Argonne National Laboratory, 9700 South Cass Avenue, Argonne, IL 60439.

This work was funded by the Department of Energy's Office of Vehicle and Engine Research and Development. Argonne National Laboratory is operated by the University of Chicago for DOE. 

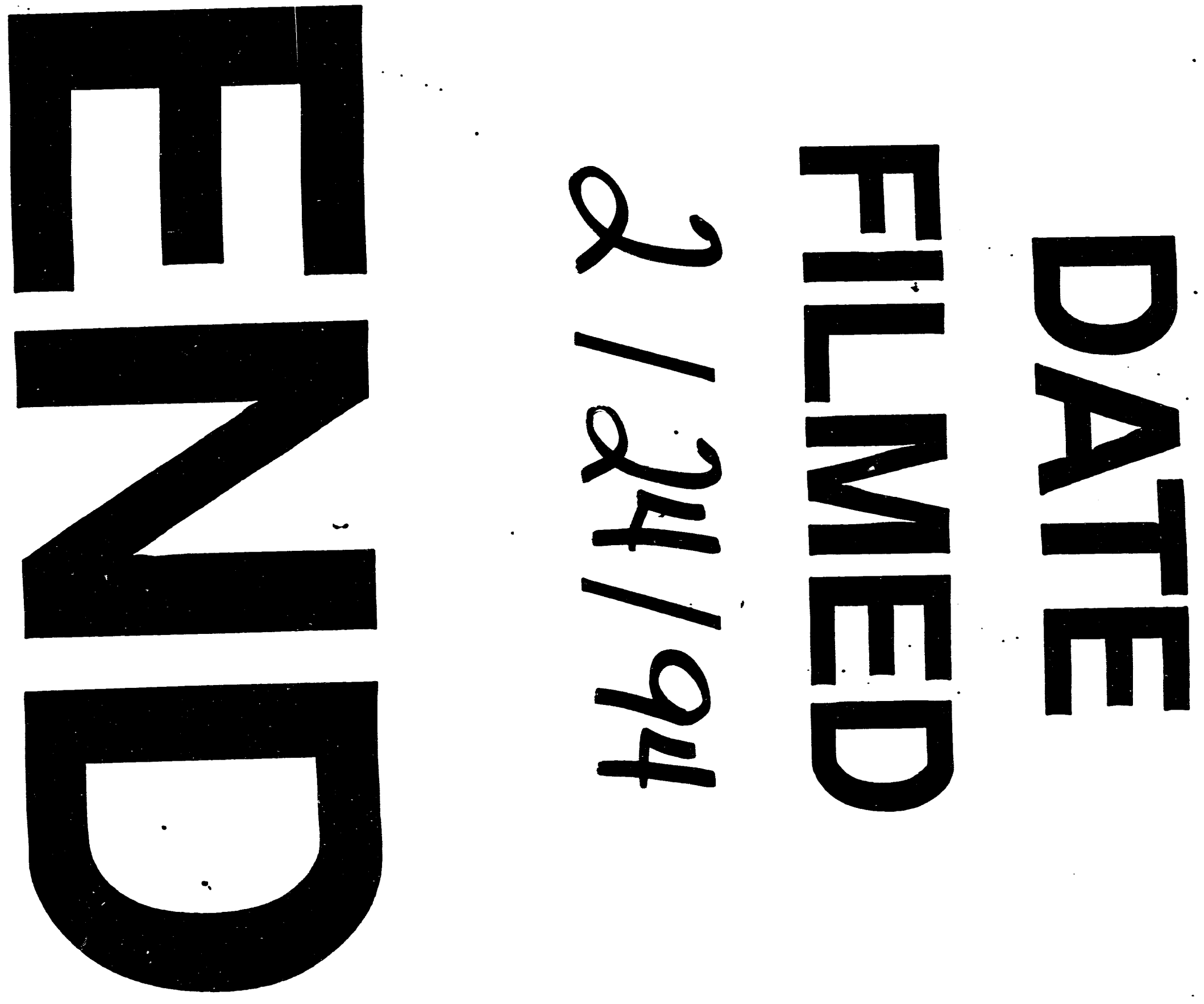
